\title{
What are the Differences in Injury Proportions Between Different Populations of Runners? A Systematic Review and Meta-Analysis
}

\author{
Bas Kluitenberg $^{1} \cdot{\text { Marienke van } \text { Middelkoop }^{2} \cdot \text { Ron Diercks }^{1} \cdot \text { Henk van der Worp }}^{1}$
}

Published online: 8 April 2015

(c) The Author(s) 2015. This article is published with open access at Springerlink.com

\begin{abstract}
Background Many runners suffer from injuries. No information on high-risk populations is available so far though.

Objectives The aims of this study were to systematically review injury proportions in different populations of runners and to compare injury locations between these populations.

Data Sources An electronic search with no date restrictions was conducted up to February 2014 in the PubMed, Embase, SPORTDiscus and Web of Science databases. The search was limited to original articles written in English. The reference lists of the included articles were checked for potentially relevant studies.

Study Eligibility Criteria Studies were eligible when the proportion of running injuries was reported and the participants belonged to one or more homogeneous populations of runners that were clearly described. Study
\end{abstract}

Electronic supplementary material The online version of this article (doi:10.1007/s40279-015-0331-x) contains supplementary material, which is available to authorized users.

Bas Kluitenberg

b.kluitenberg@umcg.nl

1 Center for Sports Medicine, University Medical Center Groningen, University of Groningen, Hanzeplein 1, 9713 GZ Groningen, The Netherlands

2 Department of General Practice, Erasmus MC Medical University, Wytemaweg 80, 3015 CN Rotterdam, The Netherlands selection was conducted by two independent reviewers, and disagreements were resolved in a consensus meeting.

Study Appraisal and Synthesis Methods Details of the study design, population of runners, sample size, injury definition, method of injury assessment, number of injuries and injury locations were extracted from the articles. The risk of bias was assessed with a scale consisting of eight items, which was specifically developed for studies focusing on musculoskeletal complaints.

Results A total of 86 articles were included in this review. Where possible, injury proportions were pooled for each identified population of runners, using a random-effects model. Injury proportions were affected by injury definitions and durations of follow-up. Large differences between populations existed. The number of medical-attention injuries during an event was small for most populations of runners, except for ultra-marathon runners, in which the pooled estimate was $65.6 \%$. Time-loss injury proportions between different populations of runners ranged from $3.2 \%$ in cross-country runners to $84.9 \%$ in novice runners. Overall, the proportions were highest among short-distance track runners and ultra-marathon runners.

Limitations The results were pooled by stratification of studies according to the population, injury definition and follow-up/recall period; however, heterogeneity was high. Conclusions Large differences in injury proportions between different populations of runners existed. Injury proportions were affected by the duration of follow-up. A U-shaped pattern between the running distance and the time-loss injury proportion seemed to exist. Future prospective studies of injury surveillance are highly recommended to take running exposure and censoring into account. 


\section{Key Points}

Many studies have examined injury occurrence among runners; however, no information on highrisk populations is available so far.

Large differences in injury proportions existed between different populations of runners.

Injury proportions were affected by the duration of follow-up. Overall, however, time-loss injury proportions were highest among short-distance track runners and ultra-marathon runners.

\section{Introduction}

Injuries are a major problem among runners. Except for previous injuries, no consistent risk factors for running injuries have been found [1]. Running injuries often lead to a reduction in running activity and generally require a long time for recovery [2]. Moreover, injuries are frequently mentioned as a reason for quitting running [3, 4]. Various studies have examined injury proportions (i.e. both incidence proportions and prevalence rates) among runners. The research is, however, characterized by conflicting results, with injury proportions varying between 1.4 [5] and $94.4 \%$ [6]. Several reasons, such as the injury definition, method of injury assessment, study design and follow-up time, form the basis for this lack of consensus. Likewise, the type of runners included in the study sample may play an important role in these conflicting results [7]. It is plausible that injury occurrence and injury type vary between different populations of runners [8]. These differences may explain the large variance in injury proportions observed in running research.

The four-stage injury prevention model developed by Van Mechelen et al. is often used to guide injury prevention research [9]. The first step in this model is to establish the extent of the problem (i.e. the injury incidence). Thereafter, the aetiology of injuries should be studied, and preventive measures can subsequently be introduced. To examine the effectiveness of these interventions, the first stage of the model is repeated [9]. The large variance in injury proportions reported in the literature makes it difficult to determine the extent of the problem. From this perspective, greater insight into the injury susceptibility of different populations of runners may identify specific populations that are at increased risk of sustaining a specific type of injury. This information can be used to assess risk factors for specific high-risk populations, which can be used to develop preventive measures for these populations. A systematic review of the most common injuries in runners demonstrated that injury patterns differed between ultra-marathon runners and runners participating over shorter distances [8]. Until now, however, no systematic review has provided indepth information on population-specific injury proportions. The primary purpose of this article, therefore, was to systematically review injury proportions in different populations of runners. The secondary objective was to examine differences in injury location between these populations.

\section{Methods}

\subsection{Eligibility Criteria and Study Selection}

A search with no date restrictions was conducted in the PubMed, Embase, SPORTDiscus and Web of Science databases up to 20 February 2014. The search strategy, as presented in Electronic Supplementary Material Appendix $\mathrm{S} 1$, was used to identify articles about injuries in runners. The search was limited to original articles written in English. Studies that met the following eligibility criteria were included in the review:

1. The study design was a prospective cohort study; retrospective cohort study; cross-sectional study; or randomized, controlled trial.

2. The subject of the study was injuries in runners.

3. Injury proportions (incidence proportion or prevalence) were reported.

4. The populations from which the participants were drawn were clearly described.

5. The participants belonged to one or more homogeneous populations of runners, and injury proportions were presented for these different populations separately.

6. Running was the main sport activity of the participants (i.e. not triathlon participants, physical education students or military recruits).

After removal of duplicate studies, all study titles and abstracts were screened by two independent reviewers (BK and HW). All articles of interest were retrieved in full text and evaluated for eligibility by the same independent reviewers. The reference lists of the included articles were checked for other potentially relevant articles that had not been not identified in the electronic search strategy.

Disagreements were resolved in a consensus meeting. On the basis of these articles, nine different populations of interest were defined in which studies were classified (Table 1). 
Table 1 Definitions of different populations of runners used to classify the articles

\begin{tabular}{ll}
\hline Population & Definition \\
\hline Track: sprinters & Track athletes competing in distances of up to $400 \mathrm{~m}$ \\
Track: middle-distance runners & Track athletes competing in distances of $800-3000 \mathrm{~m}$ \\
Track: long-distance runners & Track athletes competing in 5000 or $10,000 \mathrm{~m}$ races \\
Novice runners & Runners with no regular running experience within the previous year \\
Recreational runners & Non-competitive runners or runners participating in road races shorter than $10 \mathrm{~km}$ \\
Cross-country runners & Runners competing in cross-country races \\
Road: long-distance runners & Runners competing in races of between $10 \mathrm{~km}$ and less than a marathon \\
Marathon runners & Runners competing in a marathon \\
Ultra-marathon runners & Runners competing in races longer than a marathon \\
\hline
\end{tabular}

\subsection{Risk of Bias Assessment and Data Extraction}

Risk of bias (ROB) was assessed with a list specifically developed for assessing ROB in studies with different designs focusing on musculoskeletal complaints. The list was developed by van der Windt et al. and was made specific for epidemiology of running injuries by Nielsen et al. $[10,11]$. The criteria for the ROB assessment are presented in Electronic Supplementary Material Appendix S2. All items were scored as positive $(+)$ or negative $(-)$ by two independent review authors (BK and HW). When no clear information regarding the item was given or when it was unclear whether the ROB criteria for an item was met, the item was scored as negative. The results of both reviewers' ROB assessments were compared, and disagreements were resolved in a consensus meeting. The total ROB score for each study was calculated by counting the number of items that were scored positively, expressed as a percentage of all items. Articles with a ROB score $\geq 50 \%$ were considered as having a low ROB.

From the included articles, descriptive data on the study design, study population, sample size, injury definition, method of injury assessment, number of injuries and injury locations were extracted by one reviewer (BK). When a study reported information for multiple populations of runners, data for each population were extracted separately. Injury definitions were categorized into time-loss injuries, pain-related injuries and medical-attention injuries. When the injury hampered training for at least one day, it was categorized as a time-loss injury. Pain-related injuries were those where running-related pain was assessed regardless of the consequences for running participation (frequency/intensity/duration) and performance. Studies in which runners visited a medical aid station or injuries were reported to a clinician were considered medical-attention injuries.

Details of injury proportions with corresponding followup or recall times were acquired from cohort studies. Only injury proportions were extracted from cross-sectional studies. For randomized, controlled trials, the proportion of injuries in the total group of participants was used in the analysis. When information on the anatomical location of the injuries was reported, these data were extracted as well.

\subsection{Data Analyses}

A meta-analysis was conducted on studies that reported the injury proportion for overall injuries (i.e. studies reporting only a specific injury were excluded from the meta-analysis). First, within each population of runners, studies were categorized into four groups on the basis of the follow-up or recall period during which injuries were monitored. Studies were grouped into studies during an event, studies with a short follow-up/recall period (6-15 weeks), studies with a 1-year follow-up/recall period (11-13 months) and studies with a follow-up/recall period longer than a year. Next, studies with a similar injury definition (i.e. medical-attention, pain-related or time-loss) were grouped. When possible, injury proportions were pooled for each subgroup (same population, same follow-up/recall period and same injury definition) to reduce heterogeneity. R statistics (version 3.1.2; R Core Team 2014) [12] were used to calculate variances around the estimated incidence proportions. The $\mathrm{R}$ package meta was used to calculate pooled estimates for all subgroups [13]. Because heterogeneity between studies was expected, random-effect models were used for all analyses. To calculate heterogeneity between studies, $I^{2}$ and $\tau^{2}$ statistics were used. $I^{2}$ is an index of heterogeneity and represents the percentage of the total variance that is due to variation between studies, while $\tau^{2}$ expresses variance between studies in a random-effects metaanalysis [14]. A meta-analysis was first conducted on all studies, followed by a sensitivity analysis on the studies with a low ROB (score $\geq 50 \%$ ).

Site-specific injury proportions were calculated from the extracted data for different anatomical regions. Injuries were categorized into nine different anatomical regions, which were based on another systematic review on injury incidence among long-distance runners (hip/pelvis, upper leg, knee, lower leg, ankle, foot, lower extremity not possible to categorize, not lower extremity and other sites) 
[15]. For each anatomical location, a weighted average injury proportion was calculated per population of runners.

\section{Results}

\subsection{Full-Text Selection}

After examination of 3320 titles and abstracts, 217 potentially relevant full-text articles were retrieved. After review of the full texts, 143 articles were excluded. The reference lists of the 74 remaining articles were checked, and 12 articles were added to the review [5, 16-26]. Hence a total of 86 articles were included in the review (Fig. 1).

\subsection{Study Characteristics}

Fourteen studies presented injury proportions for multiple populations of runners separately [17, 18, 27-38] and were classified into multiple populations of runners (Table 2).
For track runners, injury proportions were reported for sprinters in 11 studies [17, 18, 27-32, 39-41], nine studies examined middle-distance runners [17, 18, 27-33] and six studies looked at injury proportions among long-distance track runners [17, 29-33]. Cross-country runners were studied in 21 articles [5, 6, 16, 19-22, 24, 26, 42-53], and long-distance runners were studied in 14 articles [3, 23, 34-38, 54-60]. Most studies were conducted among marathon runners $(N=23)$ [29-31, 34-36, 38, 61-74], while seven studies focused on ultra-marathon runners [37, 75-80]. Thirteen studies monitored injury occurrence among novice runners [25, 81-92]. The smallest number of studies was conducted in recreational non-competitive runners $(N=4)$ [7, 93-95].

Of the 86 included articles, 51 were prospective cohort studies [5-7, 16, 20-22, 24-31, 35, 36, 39-47, 49-53, 60, 69-72, 75-79, 81, 84-87, 91, 92, 95-97]. Of these, seven also included a retrospective injury proportion $[6,46,50$, 51, 53, 71, 72]. Twenty-four studies solely used a retrospective cohort design [3, 17-19, 23, 33, 34, 37, 38, 54-58,

Fig. 1 Flow chart of the article selection process

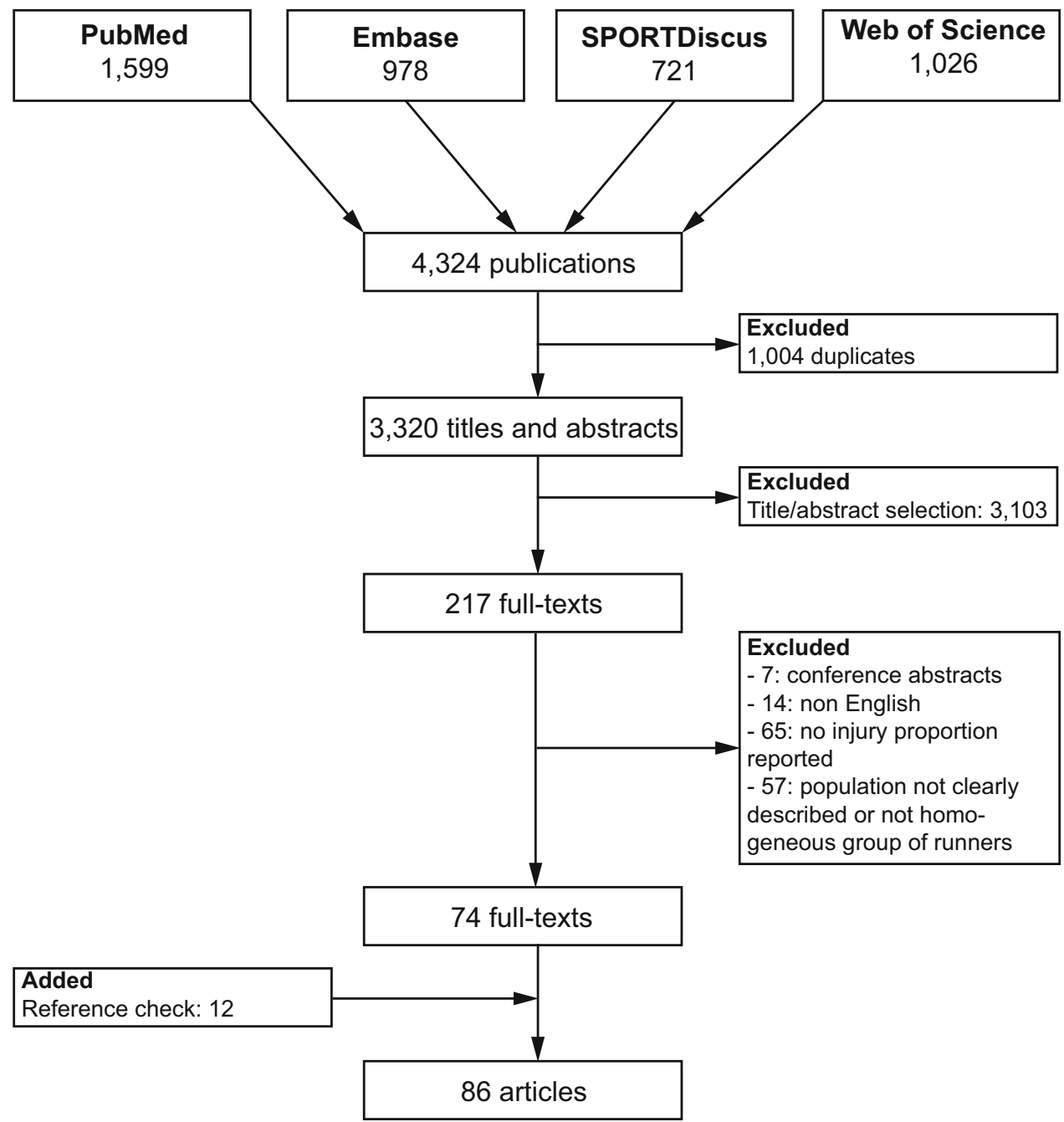


Table 2 Study characteristics and injury proportions for the different populations of runners

\begin{tabular}{|c|c|c|c|c|c|c|}
\hline Population and studies & Design & $\begin{array}{l}\text { Injury } \\
\text { definitions }\end{array}$ & Injury data & $\begin{array}{l}\text { Injury } \\
\text { proportions (\%) }\end{array}$ & Time period & $\begin{array}{l}\text { ROB } \\
(\%)\end{array}$ \\
\hline \multicolumn{7}{|l|}{ Track: sprinters } \\
\hline Longo et al. [32] & CS & PRI & AT in $29 / 41$ & 70.7 & - & 62.5 \\
\hline Alonso et al. [29] ${ }^{\mathrm{a}}$ & $\mathrm{PC}$ & MA & $\begin{array}{l}\text { (1) TLI in } 8 / 327 \\
\text { (2) PRI in } 17 / 327^{b}\end{array}$ & $\begin{array}{l}\text { (1) } 2.4 \\
\text { (2) } 5.2\end{array}$ & $\begin{array}{l}9 \text { days } \\
\quad(\text { event })\end{array}$ & 75.0 \\
\hline Alonso et al. [30] & $\mathrm{PC}$ & MA & TLI in $16 / 412$ & 3.9 & $\begin{array}{l}9 \text { days } \\
\text { (event) }\end{array}$ & 62.5 \\
\hline Alonso et al. [31] $]^{\mathrm{a}}$ & $\mathrm{PC}$ & MA & $\begin{array}{l}\text { (1) TLI in } 12 / 324 \\
\text { (2) PRI in } 31 / 324^{b}\end{array}$ & $\begin{array}{l}\text { (1) } 3.7 \\
\text { (2) } 9.6\end{array}$ & $\begin{array}{l}9 \text { days } \\
\text { (event) }\end{array}$ & 75.0 \\
\hline Bennell et al. [17] & $\mathrm{RC}$ & MA & $4 \mathrm{SFs}$ in $2 / 6$ & 33.3 & Lifetime & 62.5 \\
\hline Yeung et al. $[40]^{\mathrm{a}}$ & $\mathrm{PC}$ & TLI & $\begin{array}{l}\text { (1) Injury in } 25 / 44^{b} \\
\text { (2) } 12 \text { hamstring injuries in } 8 / 44\end{array}$ & $\begin{array}{l}\text { (1) } 56.8 \\
\text { (2) } 18.2\end{array}$ & 11 months & 87.5 \\
\hline $\begin{array}{l}\text { Lysholm and } \\
\text { Wiklander [27] }^{\mathrm{a}}\end{array}$ & $\mathrm{PC}$ & TLI & 21 injuries in $13 / 19^{\mathrm{b}} ; 5.8 / 1000 \mathrm{~h}$ & 68.4 & 1 year & 75.0 \\
\hline D’Souza $[18]^{\mathrm{a}}$ & $\mathrm{RC}$ & TLI & Injury in $27 / 40^{\mathrm{b}}$ & 67.5 & 1 year & 37.5 \\
\hline Bennell et al. [28] & $\mathrm{PC}$ & TLI & 4 SFs in $2 / 16$ & 12.5 & 1 year & 87.5 \\
\hline Sugiura et al. [39] & $\mathrm{PC}$ & TLI & Hamstring injury in $6 / 30$ & 20.0 & 1 year & 75.0 \\
\hline Jacobsson et al. $[41]^{\mathrm{a}}$ & $\mathrm{PC}$ & TLI & 122 injuries in $50 / 77^{\mathrm{b}}$ & 64.9 & 1 year & 75.0 \\
\hline \multicolumn{7}{|c|}{ Track: middle-distance runners } \\
\hline Longo et al. [32] & $\mathrm{CS}$ & PRI & AT in $22 / 32$ & 68.8 & - & 62.5 \\
\hline Alonso et al. [29] ${ }^{\mathrm{a}}$ & $\mathrm{PC}$ & MAI & $\begin{array}{l}\text { (1) TLI in } 5 / 172 \\
\text { (2) PRI in } 16 / 172^{b}\end{array}$ & $\begin{array}{l}\text { (1) } 2.9 \\
\text { (2) } 9.3\end{array}$ & $\begin{array}{l}9 \text { days } \\
\text { (event) }\end{array}$ & 75.0 \\
\hline Alonso et al. [30] & $\mathrm{PC}$ & MAI-TLI & TLI in $7 / 202$ & 3.5 & $\begin{array}{l}9 \text { days } \\
\text { (event) }\end{array}$ & 62.5 \\
\hline Alonso et al. $[31]^{\mathrm{a}}$ & $\mathrm{PC}$ & MAI & $\begin{array}{l}\text { (1) TLI in } 8 / 154 \\
\text { (2) PRI in } 26 / 154^{b}\end{array}$ & $\begin{array}{l}\text { (1) } 5.2 \\
\text { (2) } 16.9\end{array}$ & $\begin{array}{l}9 \text { days } \\
\quad(\text { event })\end{array}$ & 75.0 \\
\hline Bennell at al. [17] & $\mathrm{RC}$ & MAI & $8 \mathrm{SFs}$ in $5 / 20$ & 25.0 & Lifetime & 62.5 \\
\hline $\begin{array}{l}\text { Lysholm and } \\
\text { Wiklander [27] }\end{array}$ & $\mathrm{PC}$ & TLI & 16 injuries in $10 / 13^{\mathrm{b}} ; 5.6 / 1000 \mathrm{~h}$ & 76.9 & 1 year & 75.0 \\
\hline D'Souza $[18]^{\mathrm{a}}$ & $\mathrm{RC}$ & TLI & Injury in $15 / 27^{\mathrm{b}}$ & 55.6 & 1 year & 37.5 \\
\hline Bennell et al. [28] & $\mathrm{PC}$ & TLI & 9 SFs in $8 / 35$ & 22.9 & 1 year & 87.5 \\
\hline $\begin{array}{r}\text { Fredericson } \\
\text { et al. [33] }\end{array}$ & $\mathrm{RC}$ & N/A & $\mathrm{SF}$ in $20 / 86$ & 23.3 & Lifetime & 12.5 \\
\hline \multicolumn{7}{|c|}{ Track: long-distance runners } \\
\hline Longo et al. [32] & CS & PRI & AT in $27 / 44$ & 61.4 & - & 62.5 \\
\hline Alonso et al. [29] ${ }^{\mathrm{a}}$ & $\mathrm{PC}$ & MAI & $\begin{array}{l}\text { (1) TLI in } 6 / 101 \\
\text { (2) PRI in } 17 / 10^{b}\end{array}$ & $\begin{array}{l}\text { (1) } 5.9 \\
\text { (2) } 16.8\end{array}$ & $\begin{array}{l}9 \text { days } \\
\text { (event) }\end{array}$ & 75.0 \\
\hline Alonso et al. [30] & $\mathrm{PC}$ & MAI-TLI & TLI in $8 / 130$ & 6.2 & $\begin{array}{l}9 \text { days } \\
\text { (event) }\end{array}$ & 62.5 \\
\hline Alonso et al. $[31]^{\mathrm{a}}$ & $\mathrm{PC}$ & MAI & $\begin{array}{l}\text { (1) TLI in } 10 / 105 \\
\text { (2) PRI in } 15 / 105^{b}\end{array}$ & $\begin{array}{l}\text { (1) } 9.5 \\
\text { (2) } 14.3\end{array}$ & $\begin{array}{l}9 \text { days } \\
\text { (event) }\end{array}$ & 75.0 \\
\hline Bennell et al. [17] & $\mathrm{RC}$ & MAI & $18 \mathrm{SFs}$ in $6 / 10$ & 60.0 & Lifetime & 62.5 \\
\hline $\begin{array}{r}\text { Fredericson } \\
\text { et al. [33] }\end{array}$ & $\mathrm{RC}$ & N/A & $\mathrm{SF}$ in $57 / 188$ & 30.3 & Lifetime & 12.5 \\
\hline \multicolumn{7}{|l|}{ Novice runners } \\
\hline Thijs et al. [84] & $\mathrm{PC}$ & MAI & PFP in $17 / 102$ & 16.7 & 10 weeks & 62.5 \\
\hline $\begin{array}{l}\text { Ghani Zadeh Hesar } \\
\text { et al. [85] }\end{array}$ & $\mathrm{PC}$ & MAI & Injury in $27 / 131$ & 20.6 & 10 weeks & 62.5 \\
\hline
\end{tabular}


Table 2 continued

\begin{tabular}{|c|c|c|c|c|c|c|}
\hline Population and studies & Design & $\begin{array}{l}\text { Injury } \\
\text { definitions }\end{array}$ & Injury data & $\begin{array}{l}\text { Injury } \\
\text { proportions }(\%)\end{array}$ & Time period & $\begin{array}{l}\mathrm{ROB} \\
(\%)\end{array}$ \\
\hline \multirow[t]{3}{*}{ Buist et al. $[82,83]^{\mathrm{a}}$} & \multirow[t]{3}{*}{$\mathrm{RCT}$} & \multirow[t]{3}{*}{ TLI } & (1) Injury in $48 / 236 ; 38 / 1000 \mathrm{~h}$ & (1) 20.3 & (1) 8 weeks & 62.5 \\
\hline & & & (2) Injury in $52 / 250 ; 30 / 1000 \mathrm{~h}$ & (2) 20.8 & (2) 13 weeks & 75.0 \\
\hline & & & (3) Overall: injury in $100 / 486^{\mathrm{b}} ; 33 / 1000 \mathrm{~h}$ & (3) 20.6 & & \\
\hline \multirow{3}{*}{$\begin{array}{l}\text { Bredeweg } \\
\text { et al. }[88-90]^{\mathrm{a}}\end{array}$} & \multirow[t]{3}{*}{$\mathrm{RCT}$} & \multirow[t]{3}{*}{ TLI } & \multirow[t]{3}{*}{ Injury in $58 / 362^{\mathrm{b}} ; 32 / 1000 \mathrm{~h}$} & \multirow[t]{3}{*}{16.0} & \multirow[t]{3}{*}{9 weeks } & 62.5 \\
\hline & & & & & & 75.0 \\
\hline & & & & & & 50.0 \\
\hline Thijs et al. [87] & PC & MAI & PFP in $1.6 / 77$ & 20.8 & 10 weeks & 75.0 \\
\hline \multirow{2}{*}{$\begin{array}{l}\text { Van Ginckel } \\
\text { et al. }[86]^{\mathrm{a}}\end{array}$} & \multirow[t]{2}{*}{$\mathrm{PC}$} & \multirow[t]{2}{*}{ TLI } & (1) Injury in $69 / 129^{\mathrm{b}}$ & (1) 53.5 & \multirow[t]{2}{*}{10 weeks } & \multirow[t]{2}{*}{75.0} \\
\hline & & & (2) AT in $10 / 129$ & (2) 7.8 & & \\
\hline Nielsen et al. $[92]^{\mathrm{a}}$ & $\mathrm{PC}$ & TLI & Injury in $13 / 58^{\mathrm{b}}$ & 22.4 & 10 weeks & 75.0 \\
\hline $\begin{array}{l}\text { Nielsen } \\
\text { et al. }[25,91]^{\mathrm{a}}\end{array}$ & $\mathrm{PC}$ & TLI & Injury in $254 / 930^{\mathrm{b}}$ & 27.3 & 1 year & 75.0 \\
\hline Bovens et al. $[81]^{\mathrm{a}}$ & $\mathrm{PC}$ & TLI & 174 injuries in $62 / 73$ runners $^{\mathrm{b}}$ & 84.9 & 18 months & 50.0 \\
\hline \multicolumn{7}{|l|}{ Recreational runners } \\
\hline Lopes et al. [93] & CS & PRI & Injury in $227 / 1049$ & 21.6 & - & 62.5 \\
\hline \multirow[t]{2}{*}{ Buist et al. $[7]^{\mathrm{a}}$} & \multirow[t]{2}{*}{$\mathrm{PC}$} & (1) TLI & (1) TLI in $163 / 629^{\mathrm{b}} ; 30 / 1000 \mathrm{~h}$ & (1) 25.9 & \multirow[t]{2}{*}{8 weeks } & \multirow[t]{2}{*}{50.0} \\
\hline & & (2) PRI & (2) PRI in $217 / 629^{\mathrm{b}}$ & (2) 34.5 & & \\
\hline $\begin{array}{l}\text { Hespanhol Junior } \\
\text { et al. }[95]^{\mathrm{a}}\end{array}$ & $\mathrm{PC}$ & TLI & 84 injuries in $60 / 191^{\mathrm{b}} ; 10 / 1000 \mathrm{~h}$ & 31.4 & 12 weeks & 75.0 \\
\hline $\begin{array}{l}\text { Hespanhol Junior } \\
\text { et al. }[94]^{\mathrm{a}}\end{array}$ & $\mathrm{RC}$ & TLI & Injury in $110 / 200^{\mathrm{b}}$ & 55.0 & 1 year & 62.5 \\
\hline \multicolumn{7}{|l|}{ Cross-country runners } \\
\hline \multirow[t]{3}{*}{ Bennett et al. [53] ${ }^{\mathrm{a}}$} & (1) $\mathrm{RC}$ & \multirow[t]{3}{*}{ PRI } & (1a) Injury in $26 / 77$ & (1a) 33.8 & (a) 1 month & \multirow[t]{3}{*}{12.5} \\
\hline & \multirow[t]{2}{*}{ (2) $\mathrm{PC}$} & & (1b) Injury in $56 / 77^{\mathrm{b}}$ & (1b) 72.2 & (b) 1 year & \\
\hline & & & (2c) Injury in $26 / 59^{b}$ & (2c) 44.1 & (c) 1 season & \\
\hline Beachy et al. $[16]^{\mathrm{a}}$ & PC & (1) PRI & (1) 843 PRIs in $610 / 1288^{b}$ & (1) 47.4 & 1 season & 75.0 \\
\hline & & (2) TLI & (2) 272 TLIs in $197 / 1288^{b}$ & (2) 15.3 & & \\
\hline Reinking $[6]^{\mathrm{a}}$ & (1) $\mathrm{RC}$ & (a) PRI & (1a) PRI in $17 / 18^{\mathrm{b}}$ & (1a) 94.4 & (1) Lifetime & 62.5 \\
\hline & (2) $\mathrm{PC}$ & (b) TLI & (1b) TLI in $12 / 18^{b}$ & (1b) 66.7 & (2) 1 season & \\
\hline & & & (2a) PRI in $9 / 18^{b}$ & (2a) 50.0 & & \\
\hline & & & (2b) TLI in $3 / 18^{\mathrm{b}}$ & (2b) 16.7 & & \\
\hline Plisky et al. [49] & $\mathrm{PC}$ & PRI & 17 MTSSs in $16 / 105 ; 2.8 / 1000$ AEs & 15.2 & 1 season & 100 \\
\hline Finnoff et al. [52] & $\mathrm{PC}$ & PRI & Knee pain in $3 / 57$ & 5.3 & 1 season & 62.5 \\
\hline Garrick and & $\mathrm{PC}$ & TLI & Injury in $50 / 167^{\mathrm{b}}$ & 29.9 & 1 season & 62.5 \\
\hline Requa $[20,21]^{\mathrm{a}}$ & & & & & & 75.0 \\
\hline $\begin{array}{l}\text { Chandy and } \\
\text { Grana }[5]^{\mathrm{a}}\end{array}$ & PC & TLI & Injury in $31 / 2278^{\mathrm{b}}$ & 1.4 & 1 season & 75.0 \\
\hline Rauh et al. $[42]^{\mathrm{a}}$ & PC & TLI & $\begin{array}{l}1622 \text { injuries in } 927 / 3233^{\mathrm{b}} ; 13.1 / 1000 \text { AEs; } 8.7 \\
\text { new injuries/1000 AEs }\end{array}$ & 28.7 & 1 season & 87.5 \\
\hline Rauh et al. $[44,45]^{\mathrm{a}}$ & $\mathrm{PC}$ & TLI & 316 injuries in $162 / 421^{\mathrm{b}} ; 10.4 / 1000 \mathrm{AEs}$ & 38.5 & 1 season & 87.5 \\
\hline & & & & & & 62.5 \\
\hline Reinking and & (1) $\mathrm{RC}$ & TLI & (1) Lower leg injury in $33 / 63$ & (1) 52.4 & (1) HS career & 50.0 \\
\hline Hayes [46] & (2) $\mathrm{PC}$ & & (2) Lower leg injury in $10 / 32$ & (2) 31.3 & (2) 1 season & \\
\hline Reinking et al. $[51]^{\mathrm{a}}$ & (1) $\mathrm{RC}$ & TLI & (1) Injury in $103 / 125^{\mathrm{b}}$ & (1) 82.4 & (1) Lifetime & 50.0 \\
\hline & (2) $\mathrm{PC}$ & & (2) Injury in $45 / 93^{\mathrm{b}}$ & (2) 48.4 & (2) 1 season & \\
\hline Grana $[22]^{\mathrm{a}}$ & PC & TLI & Injury in $9 / 486^{\mathrm{b}}$ & 1.9 & 1 year & 25.0 \\
\hline Shively et al. $[26]^{\mathrm{a}}$ & $\mathrm{PC}$ & TLI & Injury in $9 / 576^{\mathrm{b}}$ & 1.6 & 1 year & 50.0 \\
\hline $\begin{array}{l}\text { McLain and } \\
\text { Reynolds }[24]^{\mathrm{a}}\end{array}$ & $\mathrm{PC}$ & TLI & Injury in $10 / 94^{\mathrm{b}}$ & 10.6 & 1 year & 75.0 \\
\hline
\end{tabular}


Table 2 continued

\begin{tabular}{|c|c|c|c|c|c|c|}
\hline Population and studies & Design & $\begin{array}{l}\text { Injury } \\
\text { definitions }\end{array}$ & Injury data & $\begin{array}{l}\text { Injury } \\
\text { proportions (\%) }\end{array}$ & Time period & $\begin{array}{l}\text { ROB } \\
(\%)\end{array}$ \\
\hline Laker et al. [48] & CS & N/A & SF in $9 / 25$ & 36.0 & - & 25.0 \\
\hline Bennett et al. [43] & $\mathrm{PC}$ & N/A & MTSS in $15 / 125$ & 12.0 & 1 season & 75.0 \\
\hline Reinking et al. [50] & $\begin{array}{l}\text { (1) } \mathrm{RC} \\
\text { (2) } \mathrm{PC}\end{array}$ & N/A & $\begin{array}{l}\text { (1) Injury in } 60 / 88 \\
\text { (2) Injury in } 26 / 67\end{array}$ & $\begin{array}{l}\text { (1) } 68.2 \\
\text { (2) } 38.8\end{array}$ & $\begin{array}{l}\text { (1) Running } \\
\text { career } \\
\text { (2) } 1 \text { season }\end{array}$ & 25.0 \\
\hline Kelsey et al. [47] & $\mathrm{PC}$ & N/A & SF in $18 / 127$ & 14.2 & 2 years & 50.0 \\
\hline Eickhoff et al. [19] & $\mathrm{RC}$ & N/A & $\begin{array}{l}\text { (1) Injury in } 101 / 164 \\
\text { (2) MTSS in } 41 / 164\end{array}$ & $\begin{array}{l}\text { (1) } 61.6 \\
\text { (2) } 25.0\end{array}$ & Lifetime & 37.5 \\
\hline \multicolumn{7}{|c|}{ Road: long-distance runners } \\
\hline $\begin{array}{l}\text { Nicholl and } \\
\text { Williams [35] }\end{array}$ & $\mathrm{PC}$ & MAI & 46 first-aid stops for 44 injuries in $41 / 1140^{b}$ & 3.6 & 1 day (event) & 62.5 \\
\hline Yeung et al. $[36]^{\mathrm{a}}$ & $\mathrm{PC}$ & MAI & Injury in $25 / 4600^{\mathrm{b}}$ & 0.5 & 1 day (event) & 62.5 \\
\hline Pasquina et al. $[60]^{\mathrm{a}}$ & $\mathrm{PC}$ & MAI & Injury in $346 / 91,750^{\mathrm{b}}$ & 0.4 & 1 day (event) & 62.5 \\
\hline Hughes et al. [54] ${ }^{\mathrm{a}}$ & $\mathrm{RC}$ & TLI & $\begin{array}{l}\text { (1) Injury in } 725 / 1266 \\
\text { (2) Injury in } 360 / 1266^{b}\end{array}$ & $\begin{array}{l}\text { (1) } 57.3 \\
\text { (2) } 28.4\end{array}$ & $\begin{array}{l}\text { (1) } \text { N/A } \\
\text { (2) } 1 \text { day } \\
\text { (event) }\end{array}$ & 62.5 \\
\hline Koplan et al. $[23]^{\mathrm{a}}$ & $\mathrm{RC}$ & TLI & Injury in $498 / 1423^{b}$ & 35.0 & 1 year & 37.5 \\
\hline Marti et al. $[57,58]^{\mathrm{a}}$ & $\mathrm{RC}$ & TLI & $\begin{array}{l}\text { (1) PRI in } 2166 / 4786^{\mathrm{b}} \\
\text { (2) TLI in } 1372 / 4786^{\mathrm{b}} \\
\text { (3) Severe TLI in } 938 / 4786\end{array}$ & $\begin{array}{l}\text { (1) } 45.3 \\
\text { (2) } 28.7 \\
\text { (3) } 19.6\end{array}$ & 1 year & $\begin{array}{l}62.5 \\
75.0\end{array}$ \\
\hline $\begin{array}{l}\text { Jacobs and } \\
\text { Berson }[55]^{\mathrm{a}}\end{array}$ & $\mathrm{RC}$ & TLI & Injury in $210 / 451^{\mathrm{b}}$ & 46.6 & 2 years & 37.5 \\
\hline Koplan et al. $[3]^{\mathrm{a}}$ & $\mathrm{RC}$ & TLI & Injury in $281 / 535^{\mathrm{b}}$ & 52.5 & 10 years & 50.0 \\
\hline Lloyd et al. $[56]^{\mathrm{a}}$ & $\mathrm{RC}$ & TLI & Injury in $80 / 260^{\mathrm{b}}$ & 30.8 & N/A (long) & 25.0 \\
\hline Woolf et al. [59] & $\begin{array}{l}\text { (1) } \mathrm{RC} \\
\text { (2) } \mathrm{CS}\end{array}$ & N/A & $\begin{array}{l}\text { (1) Low back pain in } 327 / 436 \\
\text { (2) Low back pain in } 57 / 436\end{array}$ & $\begin{array}{l}\text { (1) } 75.0 \\
\text { (2) } 13.1\end{array}$ & $\begin{array}{l}\text { (1) Lifetime } \\
\text { (2) }-\end{array}$ & 25.0 \\
\hline $\begin{array}{l}\text { Nicholl and } \\
\text { Williams [34] }\end{array}$ & $\mathrm{RC}$ & N/A & Injury in $97 / 242$ & 40.1 & 1 week & 25.0 \\
\hline $\begin{array}{l}\text { Micklesfield } \\
\text { et al. [37] }\end{array}$ & $\mathrm{RC}$ & N/A & SF in $47 / 337$ & 13.9 & N/A & 12.5 \\
\hline Chang et al. [38] & $\mathrm{RC}$ & N/A & 487 injuries in $334 / 765$ & 43.7 & N/A & 12.5 \\
\hline Marathon runners & & & & & & \\
\hline Kretsch et al. $[63]^{\mathrm{a}}$ & $\begin{array}{l}\text { (1) } \mathrm{CS} \\
\text { (2) } \mathrm{RC}\end{array}$ & PRI-MAI & $\begin{array}{l}\text { (1) Injury in } 151 / 459 \\
\text { (2a) PRI in } 422 / 459^{b} \\
\text { (2b) MAI in } 9 / 459^{b}\end{array}$ & $\begin{array}{l}\text { (1) } 32.9 \\
\text { (2a) } 91.9 \\
\text { (2b) } 2.0\end{array}$ & $\begin{array}{l}(1)- \\
\text { (2) } 1 \text { day } \\
\text { (event) }\end{array}$ & 25.0 \\
\hline Parker et al. $[73]^{\mathrm{a}}$ & $\mathrm{RC}$ & PRI & $\begin{array}{l}\text { (1) Injury in } 186 / 374^{\mathrm{b}} \\
\text { (2) Injury in } 137 / 374^{\mathrm{b}} \\
\text { (3) Injury in } 53 / 374^{\mathrm{b}}\end{array}$ & $\begin{array}{l}\text { (1) } 49.7 \\
\text { (2) } 36.6 \\
\text { (3) } 14.2\end{array}$ & $\begin{array}{l}\text { (1) } 1 \text { year } \\
\text { (2) N/A } \\
\text { (short) } \\
\text { (3) } 1 \text { day } \\
\text { (event) }\end{array}$ & 37.5 \\
\hline $\begin{array}{l}\text { Nicholl and } \\
\text { Williams [35] }^{\mathrm{a}}\end{array}$ & $\mathrm{PC}$ & MAI & 580 first-aid stops for 534 injuries in $409 / 2289^{b}$ & 17.9 & 1 day (event) & 62.5 \\
\hline $\begin{array}{l}\text { Satterthwaite } \\
\text { et al. }[69,96]^{\mathrm{a}}\end{array}$ & $\mathrm{PC}$ & MAI & $\begin{array}{l}\text { (1a) First-aid stops in } 75 / 1219^{b} \\
\text { (1b) } 2671 \text { PRIs in } 846 / 916^{b} \\
\text { (2) } 1905 \text { PRIs in } 723 / 916\end{array}$ & $\begin{array}{l}\text { (1a) } 6.2 \\
\text { (1b) } 92.4 \\
\text { (2) } 78.9\end{array}$ & $\begin{array}{l}\text { (1) } 1 \text { day } \\
\text { (event) } \\
\text { (2) } 1 \text { week }\end{array}$ & $\begin{array}{l}75.0 \\
62.5\end{array}$ \\
\hline Yeung et al. $[36]^{\mathrm{a}}$ & $\mathrm{PC}$ & MAI & Injury in $46 / 900^{\mathrm{b}}$ & 5.1 & 1 day (event) & 62.5 \\
\hline Roberts et al. $[70]^{\mathrm{a}}$ & $\mathrm{PC}$ & MAI & 1534 injuries in $1459 / 76,714^{\mathrm{b}}$ & 1.9 & 1 day (event) & 75.0 \\
\hline $\begin{array}{l}\text { Ogwumike and } \\
\text { Adeniyi [97] }\end{array}$ & $\mathrm{PC}$ & MAI & Injury in $153 / 920^{\mathrm{b}}$ & 16.6 & 1 day (event) & 62.5 \\
\hline
\end{tabular}


Table 2 continued

\begin{tabular}{|c|c|c|c|c|c|c|}
\hline Population and studies & Design & $\begin{array}{l}\text { Injury } \\
\text { definitions }\end{array}$ & Injury data & $\begin{array}{l}\text { Injury } \\
\text { proportions (\%) }\end{array}$ & Time period & $\begin{array}{l}\text { ROB } \\
(\%)\end{array}$ \\
\hline Alonso et al. [29] ${ }^{\mathrm{a}}$ & $\mathrm{PC}$ & MAI & $\begin{array}{l}\text { (1) TLI in } 14 / 151 \\
\text { (2) PRI in } 20 / 151^{b}\end{array}$ & $\begin{array}{l}\text { (1) } 9.3 \\
\text { (2) } 13.2\end{array}$ & $\begin{array}{l}9 \text { days } \\
\text { (event) }\end{array}$ & 75.0 \\
\hline Alonso et al. [30] & $\mathrm{PC}$ & MAI-TLI & TLI in $3 / 182$ & 1.6 & $\begin{array}{l}9 \text { days } \\
\text { (event) }\end{array}$ & 62.5 \\
\hline Alonso et al. [31] ${ }^{\mathrm{a}}$ & $\mathrm{PC}$ & MAI & $\begin{array}{l}\text { (1) TLI in } 18 / 124 \\
\text { (2) PRI in } 28 / 124^{b}\end{array}$ & $\begin{array}{l}\text { (1) } 14.5 \\
\text { (2) } 22.6\end{array}$ & $\begin{array}{l}9 \text { days } \\
\text { (event) }\end{array}$ & 75.0 \\
\hline $\begin{array}{l}\text { Caldwell }[61]^{\mathrm{a}} \\
\text { Maughan and } \\
\text { Miller [62] }\end{array}$ & $\begin{array}{l}\mathrm{RC} \\
\mathrm{RC}\end{array}$ & $\begin{array}{l}\text { TLI } \\
\text { (a) TLI } \\
\text { (b) N/A }\end{array}$ & $\begin{array}{l}\text { Injury in } 68 / 116^{\mathrm{b}} \\
\text { (1a) TLI in } 216 / 497^{\mathrm{b}} \\
\text { (1b) } 358 \text { injuries in } 287 / 497 \\
\text { (2b) Injury in } 129 / 449\end{array}$ & $\begin{array}{l}58.3 \\
\text { (1a) } 43.5 \\
\text { (1b) } 57.7 \\
\text { (2b) } 28.7\end{array}$ & $\begin{array}{l}1 \text { day (event) } \\
\text { (1) N/A } \\
\text { (short) } \\
\text { (2) } 1 \text { day } \\
\text { (event) }\end{array}$ & $\begin{array}{l}25.0 \\
0\end{array}$ \\
\hline Hölmich et al. [65] ${ }^{\mathrm{a}}$ & $\mathrm{RC}$ & TLI & $\begin{array}{l}\text { Injury in } 26 / 60^{b} \\
\text { Injury in } 7 / 60^{b}\end{array}$ & $\begin{array}{l}\text { (1) } 43.3 \\
\text { (2) } 11.7\end{array}$ & $\begin{array}{l}\text { (1) } 1 \text { year } \\
\text { (2) } 1 \text { day } \\
\text { (event) }\end{array}$ & 62.5 \\
\hline $\begin{array}{l}\text { Van Middelkoop } \\
\text { et al. }[71,72]^{\mathrm{a}}\end{array}$ & $\begin{array}{l}\text { (1) } \mathrm{RC} \\
\text { (2) } \mathrm{PC}\end{array}$ & TLI & $\begin{array}{l}\text { (1a) Injury in } 397 / 725^{b} \\
\text { (2b) Injury in } 108 / 725 \\
\text { (2c) Injury in } 118 / 694^{b}\end{array}$ & $\begin{array}{l}\text { (1a) } 54.8 \\
\text { (2b) } 14.9 \\
\text { (2c) } 17.0\end{array}$ & $\begin{array}{l}\text { 1a) } 1 \text { year } \\
\text { 2b) } 1 \text { month } \\
\text { 2c) } 1 \text { day } \\
\text { (event) }\end{array}$ & $\begin{array}{l}75.0 \\
62.5\end{array}$ \\
\hline $\begin{array}{l}\text { Rasmussen } \\
\text { et al. [74] }{ }^{\mathrm{a}}\end{array}$ & $\mathrm{RC}$ & TLI & $\begin{array}{l}\text { (1) Injury in } 273 / 662^{b} \\
\text { (2) Injury in } 68 / 662^{b}\end{array}$ & $\begin{array}{l}\text { (1) } 41.2 \\
\text { (2) } 10.3\end{array}$ & $\begin{array}{l}\text { (1) } 1 \text { year } \\
\text { (2) } 1 \text { day } \\
\text { (event) }\end{array}$ & 62.5 \\
\hline Macera et al. [67] ${ }^{\mathrm{a}}$ & $\mathrm{RC}$ & TLI & $\begin{array}{l}\text { (1) Injury in } 85 / 162^{b} \\
\text { (2) Injury in } 57 / 162\end{array}$ & $\begin{array}{l}\text { (1) } 52.4 \\
\text { (2) } 35.2\end{array}$ & $\begin{array}{l}\text { (1) } 1 \text { year } \\
\text { (2) } 1 \text { month }\end{array}$ & 50.0 \\
\hline McKelvie et al. [64] ${ }^{\mathrm{a}}$ & $\mathrm{RC}$ & TLI & Injury in $103 / 126^{\mathrm{b}}$ & 81.7 & 12 weeks & 25.0 \\
\hline Jakobsen et al. [68] ${ }^{\mathrm{a}}$ & RCT & TLI & 50 injuries in $31 / 41^{\mathrm{b}}$ & 75.6 & 1 year & 62.5 \\
\hline Hölmichet al. [66] ${ }^{\mathrm{a}}$ & $\mathrm{RC}$ & TLI & Injury in $410 / 1310^{\mathrm{b}}$ & 31.3 & N/A (long) & 12.5 \\
\hline $\begin{array}{l}\text { Nicholl and } \\
\text { Williams [34] }\end{array}$ & $\mathrm{RC}$ & N/A & Injury in $203 / 312$ & 65.1 & 1 week & 25.0 \\
\hline Chang et al. [38] & $\mathrm{RC}$ & N/A & 117 injuries in $60 / 125$ & 48.0 & N/A (long) & 12.5 \\
\hline Ultra-marathon runners & & & & & & \\
\hline $\begin{array}{l}\text { Hoffman and } \\
\text { Krishnan }[80]^{\mathrm{a}}\end{array}$ & $\mathrm{RC}$ & $\begin{array}{l}\text { (1) PRI } \\
\text { (2) TLI }\end{array}$ & $\begin{array}{l}\text { (1) } 1900 \text { PRIs in } 933 / 1212^{\mathrm{b}} \\
\text { (2) TLI in } 783 / 1212^{\mathrm{b}}\end{array}$ & $\begin{array}{l}\text { (1) } 77.0 \\
\text { (2) } 64.6\end{array}$ & 1 year & 25.0 \\
\hline $\begin{array}{l}\text { Scheer and } \\
\text { Murray }[79]^{\mathrm{a}}\end{array}$ & $\mathrm{PC}$ & MAI & Clinical encounters in $39 / 69^{\mathrm{b}}$ & 56.5 & $\begin{array}{l}5 \text { days } \\
\text { (event) }\end{array}$ & 62.5 \\
\hline Hutson $[75]^{\mathrm{a}}$ & $\mathrm{PC}$ & MAI & 31 injuries in $15 / 25^{\mathrm{b}}$ & 60.0 & $\begin{array}{l}6 \text { days } \\
\text { (event) }\end{array}$ & 62.5 \\
\hline $\begin{array}{l}\text { Bishop and } \\
\text { Fallon [77] }\end{array}$ & $\mathrm{PC}$ & MAI & 36 injuries in $11 / 17^{\mathrm{b}}$ & 64.7 & $\begin{array}{l}6 \text { days } \\
\text { (event) }\end{array}$ & 75.0 \\
\hline Krabak et al. $[78]^{\mathrm{a}}$ & $\mathrm{PC}$ & MAI & 1173 injuries in $257 / 396^{\mathrm{b}} ; 65 / 1000 \mathrm{~h}$ & 64.9 & $\begin{array}{l}7 \text { days } \\
\text { (event) }\end{array}$ & 100 \\
\hline 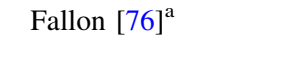 & $\mathrm{PC}$ & MAI & 64 injuries in $29 / 32^{b}$ & 90.6 & $\begin{array}{r}8.5 \text { days } \\
\text { (event) }\end{array}$ & 50.0 \\
\hline $\begin{array}{r}\text { Micklesfield } \\
\text { et al. [37] }\end{array}$ & $\mathrm{RC}$ & N/A & SF in $58 / 276$ & 21.0 & N/A & 12.5 \\
\hline
\end{tabular}

$A E$ athletic exposure, $A T$ Achilles tendinopathy, $C S$ cross-sectional study, $H S$ high school, $M A I$ medical-attention injury, MTSS medial tibial stress syndrome, N/A not available, $P C$ prospective cohort study, $P F P$ patellofemoral pain, $P R I$ pain-related injury, $R C$ retrospective cohort study, $R C T$ randomized, controlled trial, $R O B$ risk of bias, $S F$ stress fracture, $T L I$ time-loss injury

${ }^{\text {a }}$ Study included in the meta-analysis

${ }^{b}$ Data used in the meta-analysis 
$61,62,64-67,73,74,80,94]$. Five studies reported injury proportions cross-sectionally [32, 48, 59, 63, 93]-two of them retrospectively [59, 63]. From all included studies, nine reported injury incidence densities in addition to injury proportions $[7,27,42,44,49,78,83,88,95]$. Six articles reported the results of a randomized, controlled trial that reported injury occurrence [68, 82, 83, 88-90].

The follow-up periods of the included prospective cohort studies ranged from 1 day to 2 years. For retrospective cohort studies, the recall period varied from 1 day to a lifetime. A time-loss definition was used in 50 studies $[3,5-7,16,18$, 20-28, 39-42, 44-46, 51, 54-58, 61, 62, 64-68, 71, 72, 74, $80-83,86,88-92,94,95]$. Twenty studies used a medicalattention definition $[17,29-31,35,36,60,63,69,70,75-79$, $84,85,87,96,97], 11$ registered pain-related injuries [6, 7, $16,32,49,52,53,63,73,80,93]$ and in 11 studies, the injury definition was not specified $[19,33,34,37,38,43,47,48,50$, 59, 62]. All study characteristics are presented in Electronic Supplementary Material Appendix S3.

\subsection{Risk of Bias}

The results of the ROB analysis can be found in Electronic Supplementary Material Appendix S4, and total ROB scores are presented in Table 2. The overall ROB of all included articles was $57.0 \%$, ranging from 0 to $100 \%$. Twenty-one articles with an ROB score below 50 were classified as having a high ROB [18, 19, 22, 23, 33, 34, 37, $38,48,50,53,55,56,59,61-64,66,73,80]$. In general, lower ROB scores were the result of low scores on the following items of the ROB checklist: (1) the participation rate was less than $80 \%$; (2) demographics were missing/ incomplete; and (3) the main objective of the study was not to examine injury proportions.

\subsection{Meta-analyses of Injury Proportions}

Fifteen studies reported injury proportions for specific conditions only $[17,28,32,33,37,39,43,46-49,52,59$, $84,87]$, so the results of those studies could not be pooled (Table 2). The results of the meta-analysis of all studies are shown in Figs. 2, 3, 4, 5 and 6. Heterogeneity was high, as indicated by the $I^{2}$ values that exceeded $50 \%$ (Figs. 2, 3, 4, 5 and 6$)$. The results of the sensitivity analysis $(\mathrm{ROB} \geq 50)$ can be found in Electronic Supplementary Material Appendix S5. The results of the meta-analysis are described below for each follow-up/recall period and injury type.

\subsection{Medical-Attention Injuries During an Event}

Results were pooled from 22 study populations in which medical encounters during a running event were monitored (Fig. 2) [29, 31, 35, 36, 60, 63, 69, 70, 75-79, 96, 97]. The proportion of medical-attention injuries was highest in ultra-marathon runners $[65.6 \%(95 \% \mathrm{CI} 55.6-74.4)]$ and lowest in road runners [0.9\% (95\% CI $0.2-3.8)]$. The injury proportions among elite track runners varied from $7.2 \%$ (95\% CI 3.9-12.9) in sprinters to $12.8 \%(95 \% \mathrm{CI}$ 7.0-22.3) in middle-distance runners and $15.6 \%(95 \% \mathrm{CI}$ 11.2-21.2) in long-distance track runners. Medical-attention injuries during an event were not monitored in novice, recreational and cross-country runners. During a marathon race, a medical encounter was registered in $7.8 \%$ (95\% CI 2.9-19.2) of runners. All studies followed runners for a single day, with the exception of most studies in ultra-marathon runners, which followed participants during multi-day competitions. The sensitivity analysis pooled 21 study populations (see Electronic Supplementary Material Appendix S5) [29, 31, 35, 36, 60, 69, 70, 75-79, 96, 97]; this analysis revealed identical results, except for those in marathon runners, who showed an injury proportion that was slightly higher $[9.4 \%$ (95 \% CI 3.3-23.9)].

\subsubsection{Time-Loss and Pain-Related Injuries During an Event}

Five studies that included only long-distance road runners $(N=1)$ [54] and marathon runners $(N=4)[54,61,65,71$, $72,74]$ assessed the occurrence of time-loss injuries during a race. The data from these studies were pooled. Participants in short road races reported a time-loss injury proportion of $28.4 \%$ (95 \% CI 26.0-31.0). Among marathon runners, the pooled time-loss injury proportion was $20.6 \%$ (95\% CI 9.3-39.6). The sensitivity analysis of time-loss injuries during an event consisted of four studies [54, 65, 71, 72, 74]. The injury proportion among long-distance road runners was identical. The pooled injury proportion in marathon runners $(N=3)$ was $13.0 \%$ (95\% CI 8.5-19.3).

Three studies among marathon runners examined the number of pain-related injuries during an event $[63,69,73$, 96]. The pooled estimate was $73.9 \%$ (95\% CI 14.7-97.9). In the sensitivity analysis, one study remained, in which 92.4\% (95\% CI 90.4-94.0) of the participants reported a pain-related injury $[69,96]$.

\subsubsection{Time-Loss and Pain-Related Injuries During a Short Follow-Up/Recall Period}

Data were pooled from 15 study populations in which timeloss injuries were recorded during a short follow-up/recall period (Fig. 3) [5-7, 16, 20, 21, 42, 44, 45, 51, 62, 64, 82, $83,86,88-90,92,95]$. No studies were conducted in track runners (sprint, middle-distance and long-distance) with a short follow-up/recall period. The pooled injury proportion was highest in marathon runners $[64.7 \%(95 \% \mathrm{CI}$ 25.6-91.2)] and lowest in cross-country runners [19.7\% 
Study

Population $=$ Track: sprinters

Alonso et al. (2009) [29]

Alonso et al. (2012) [31]

Random effects model

Heterogeneity: $R^{2}=77.4 \%, \tau^{2}=0.1671, p=0.0355$

Population $=$ Track: middle distance runners

Alonso et al. (2009) [29]

Alonso et al. (2012) [31]

Random effects mode

Heterogeneity: $R^{2}=75.3 \%, \tau^{2}=0.1759, p=0.0441$

Population = Track: long distance runners

Alonso et al. (2009) [29]

Alonso et al. (2012) [31]

Random effects mode

Heterogeneity: $P^{2}=0 \%, \tau^{2}=0, p=0.6144$

Population = Road: long distance runners Nicholl and Williams (1983) [35]

Yeung et al. (1998) [36]

Pasquina et al. (2013) [60]

Random effects model

Heterogeneity: $P^{2}=98.9 \%, \tau^{2}=1.624, p<0.0001$

Population = Marathon runners

Nicholl and Williams (1983) [35]

Kretsch et al. (1984) [63]

Satterthwaite et al. (1996) [69], (1999) [96]

Yeung et al. (1998) [36]

Roberts (2000) [70]

Alonso et al. (2009) [29]

Alonso et al. (2012) [31]

Ogwumike and Adeniyi (2013) [97]

Random effects model

Heterogeneity: $P^{2}=99.7 \%, \tau^{2}=2.18, p<0.0001$

Population $=$ Ultra marathon runners

Hutson (1984) [75]

Fallon (1996) [ 76]

Bishop and Fallon (1999) [77]

Krabak et al. (2011) [78]

Scheer and Murray (2011) [79]

Random effects model

Heterogeneity: $P^{2}=58.9 \%, \tau^{2}=0.1173, p=0.045$
Design Injuries Participants

Injury proportion (\%)

$95 \% \mathrm{Cl}$

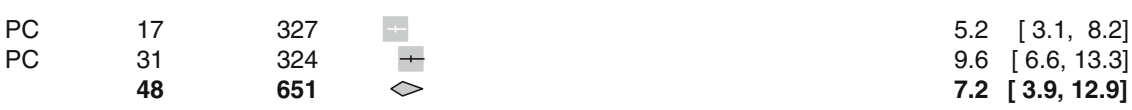

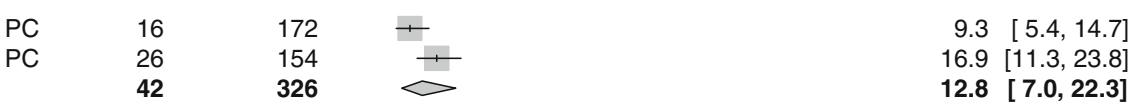

$\begin{array}{lllll}\mathrm{PC} & 17 & 101 & + & 16.8[10.1,25.6] \\ \mathrm{PC} & 15 & 105 & + & 14.3[8.2,22.5] \\ & \mathbf{3 2} & \mathbf{2 0 6} & \varnothing & \mathbf{1 5 . 6}[\mathbf{1 1 . 2 , 2 1 . 2 ]}\end{array}$

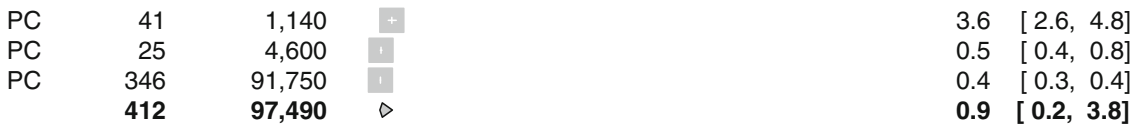

$\begin{array}{lrrrr}\text { PC } & 409 & 2,289 & \\ \text { RC } & 9 & 459 & \\ \text { PC } & 75 & 1,219 & + \\ \text { PC } & 46 & 900 & + \\ \text { PC } & 1,459 & 76,714 & \\ \text { PC } & 20 & 151 & + \\ \text { PC } & 28 & 124 & - \\ \text { PC } & 153 & 920 & +\end{array}$

$17.9[16.3,19.5]$

$2.0 \quad[0.9,3.7]$

$6.2[4.9,7.7]$

$5.1 \quad[3.8,6.8]$

$1.9[1.8,2.0]$

$13.2[8.3,19.7]$

$22.6[15.6,31.0]$

$16.6[14.3,19.2$

$7.8[2.9,19.2]$

$\begin{array}{lrr}\text { PC } & 15 & 25 \\ \text { PC } & 29 & 32 \\ \text { PC } & 11 & 17 \\ \text { PC } & 257 & 396 \\ \text { PC } & 39 & 69 \\ & 351 & 539\end{array}$

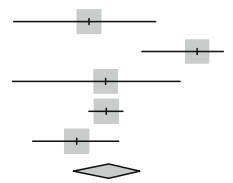

$60.0[38.7,78.9]$

$90.6[75.0,98.0]$

$64.7[38.3,85.8]$

$64.9[60.0,69.6]$

$56.5[44.0,68.4]$

$65.6[55.6,74.4]$

Fig. 2 Pooled injury proportions (\%) with $95 \%$ confidence intervals (CIs) of participants with injuries requiring medical attention during an event. $P C$ prospective cohort study, $R C$ retrospective cohort study

(95\% CI 10.9-33.1)]. In these studies, cross-country runners were often prospectively followed during a crosscountry season (of around 13 weeks). The pooled injury proportions were $26.4 \%$ (95\% CI 14.2-43.7) in novice runners and $28.0 \%(95 \% \mathrm{CI} 23.1-33.5)$ in recreational runners. Both studies among marathon runners were omitted from the sensitivity analysis [62, 64]; the other results did not change (see Electronic Supplementary Material Appendix S5).

Five studies examined the occurrence of pain-related injuries with a short follow-up/recall period (Fig. 4) [6, 7, $16,53,73]$. The data from three studies of pain-related injuries among cross-country runners were pooled, resulting in the highest pooled injury proportion [47.3\% (95 \% CI 44.6-49.9)] [6, 16, 53]. Pain-related injuries were registered in one study among recreational runners [34.5\% (95 \% CI 30.9-38.3)] [7]. In marathon runners, one study monitored pain-related injuries, with an injury proportion of $36.6 \%$ (95\% CI 31.9-41.6) [73]. The study in marathon runners [73] and a study in cross-country runners [53] were excluded from the sensitivity analysis (see Electronic Supplementary Material Appendix S5). The proportions of pain-related injuries among recreational runners were identical in the sensitivity analysis. The estimated injury proportions of pain-related injuries in cross-country runners remained similar [47.4\% (95\% CI 44.7-50.1)]. 
Study

Population $=$ Novice runners

Buist et al. (2008) [82], (2010) [83]

van Ginckel et al. (2009) [86]

Bredeweg et al. (2012) [88], (2013) [89], (2013) [90] PC

Nielsen et al. (2013) [92]

Random effects model

Heterogeneity: $l^{2}=95.7 \%, \tau^{2}=0.5871, p<0.0001$

\begin{tabular}{rrrl} 
PC & 100 & 486 & + \\
PC & 69 & 129 & \\
PC & 58 & 362 & + \\
PC & 13 & 58 & $\square$ \\
\hline
\end{tabular}

$20.6[17.1,24.4]$

$53.5[44.5,62.3]$

$16.0[12.4,20.2$

$22.4[12.5,35.3]$

$26.4[14.2,43.7]$

Population $=$ Recreational runners

Buist et al. (2010) [7]

Hespanhol Junior et al. (2013) [95]

Random effects model

Heterogeneity: $P^{2}=55.2 \%, \tau^{2}=0.02, p=0.1353$

$P C$
$P C$

Population $=$ Cross - country runners

Garrick and Requa (1978) [20], (1978) [21]

Chandy and Grana (1985) [5]

Beachy et al. (1997) [16]

Rauh et al. (2000) [42]

Rauh et al. (2006) [44], (2007) [54]

Reinking (2006) [6]

Reinking et al. (2010) [51]

Random effects model

Heterogeneity: $P^{2}=98.7 \%, \tau^{2}=0.8301, p<0.0001$

Population $=$ Marathon runners

Maughan and Miller (1983) [62]

McKelvie et al. (1985) [64]

Random effects model

Heterogeneity: $R^{2}=98 \%, \tau^{2}=1.522, p<0.0001$

$\begin{array}{lrr}\text { PC } & 50 & 167 \\ \text { PC } & 31 & 2,278 \\ \text { PC } & 197 & 1,288 \\ \text { PC } & 927 & 3,233 \\ \text { PC } & 162 & 421 \\ \text { PC } & 3 & 18 \\ \text { PC } & 45 & 93 \\ & \mathbf{1 , 4 1 5} & \mathbf{7 , 4 9 8}\end{array}$

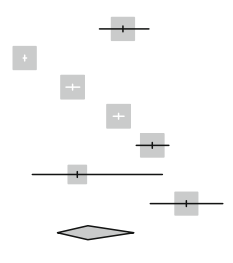

$29.9[23.1,37.5]$

$1.4 \quad[0.9,1.9]$

$15.3[13.4,17.4]$

$28.7[27.1,30.3]$

$38.5[33.8,43.3]$

$16.7 \quad[3.6,41.4]$

$48.4[37.9,59.0]$

$19.7[10.9,33.1]$

$\begin{array}{lll}\mathrm{RC} & 216 & 497 \\ \mathrm{RC} & 103 & 126 \\ & \mathbf{3 1 9} & \mathbf{6 2 3}\end{array}$

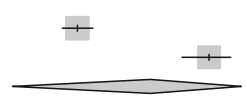

$43.5[39.1,47.9]$

$81.7[73.9,88.1]$

$64.7[24.6,91.2]$

Fig. 3 Pooled injury proportions (\%) with $95 \%$ confidence intervals (CIs) of participants with time-loss injuries during a short follow-up/recall period. $P C$ prospective cohort study, $R C$ retrospective cohort study

Study

Design Injuries Participants

Injury proportion (\%) $\quad 95 \% \mathrm{Cl}$

Population = Recreational runners

Buist et al. (2010) [7]

PC

217

217

629

629

$+$

$34.5[30.8,38.4]$

Random effects model
Heterogeneity: not applicable for a single study

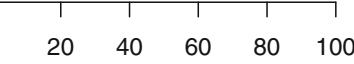

Injury proportion (\%)

Population $=$ Cross-country runners

Beachy et al. (1997) [16]

Reinking (2006) [6]

Bennett et al. (2012) [53]

PC $\quad 610 \quad 1,288$

$\begin{array}{lll}P C & 9 & 18\end{array}$

$P C$

26
645

Heterogeneity: ${ }^{2}=0 \%, \tau^{2}=0, p=0.8606$

Population $=$ Marathon runners

Parker et al. (2011) [73]

$\mathrm{RC}$

137

137

374

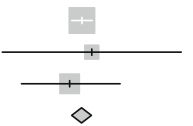

$+$
$34.5[30.9,38.3]$

$47.4[44.6,50.1]$

$50.0[26.0,74.0]$

$44.1[31.2,57.6]$

$47.3[44.6,49.9]$

$36.6[31.7,41.7]$

$36.6[31.9,41.6]$

Heterogeneity: not applicable for a single study

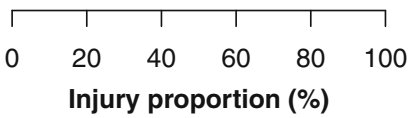

Fig. 4 Pooled injury proportions (\%) with $95 \%$ confidence intervals (CIs) of participants with pain-related injuries during a short follow-up/ recall period. $P C$ prospective cohort study, $R C$ retrospective cohort study

\subsubsection{Time-Loss Injuries During a 1-Year Follow-Up/ Recall Period}

Nineteen studies that monitored time-loss injuries for a 1-year follow-up/recall period were pooled (Fig. 5) [18,
22-27, 40, 41, 57, 58, 65, 67, 68, 71, 72, 74, 80, 91, 94]. The pooled injury proportions were highest in sprinting athletes [63.8\% (95\% CI 56.5-70.5)] and middle-distance track runners [63.9\% (95\% CI 41.4-81.6)]. No data were available for long-distance track runners. The 
Study

Population $=$ Track: sprinters

Lysholm and Wiklander (1987) [27]

D'Souza (1994) [18]

Yeung et al. (2009) [40]

Jacobsson et al. (2013) [41]

Random effects model

Heterogeneity: ${ }^{2}=0 \%, \tau^{2}=0, p=0.711$

Population $=$ Track: middle distance runners Lysholm and Wiklander (1987) [27]

D'Souza (1994) [18]

Random effects model

Heterogeneity: $P^{2}=39.4 \%, \tau^{2}=0.1893, p=0.1991$

Population $=$ Novice runners

Nielsen et al. (2013) [25], (2013) [91]

Random effects model

Heterogeneity: not applicable for a single study

Population $=$ Recreational runners

Hespanhol Junior et al. (2012) [94]

Random effects model

Heterogeneity: not applicable for a single study

Population $=$ Cross-country runners

Grana (1979) [22]

Shively et al. (1981) [26]

McLain and Reynolds (1989) [24]

Random effects model

Heterogeneity: $R^{2}=91 \%, \tau^{2}=1.139, p<0.0001$

Population $=$ Road: long distance runners Koplan et al. (1982) [23]

Marti et al. (1988) [57], (1988) [58]

Random effects model

Heterogeneity: $P^{2}=95.2 \%, \tau^{2}=0.0407, p<0.0001$

Population $=$ Marathon runners

Hölmich et al. (1988) [65]

Macera et al. (1991) [67]

Jakobsen et al. (1994) [68]

van Middelkoop et al. (2008) [71], (2008) [72]

Rasmussen et al. (2013) [74]

Random effects model

Heterogeneity: $l^{2}=89.3 \%, \tau^{2}=0.1312, p<0.0001$

Population = Ultra marathon runners

Hoffman and Krishnan (2014) [80]

Random effects model

Heterogeneity: not applicable for a single study
Design Injuries Participants

Injury proportion (\%)

$95 \% \mathrm{Cl}$
$68.4[43.4,87.4]$

$67.5[50.9,81.4]$

$56.8[41.0,71.7]$

$64.9[53.2,75.5]$

$63.8[56.5,70.5]$

$76.9[46.2,95.0]$ $55.6[35.3,74.5]$ $63.9[41.4,81.6]$

$27.3[24.5,30.3]$

$27.3[24.5,30.3]$

$55.0[47.8,62.0]$ $55.0[48.1,61.8]$

$1.9 \quad[0.9,3.5]$

$1.6 \quad[0.7,2.9]$

$10.6 \quad[5.2,18.7]$

$3.2[0.9,10.5]$

$35.0[32.5,37.5]$

$28.7[27.4,30.0]$

$31.7[25.8,38.2]$

$43.3[30.6,56.8]$

$52.5[44.5,60.4]$

$75.6[59.7,87.6]$

$54.8[51.1,58.4]$

$41.2[37.5,45.1]$

$52.0[43.1,60.8]$

$64.6[61.8,67.3]$

$64.6[61.9,67.2]$

\section{RC $\quad 783 \quad 1,212$ \\ $\mathbf{7 8 3} \quad \mathbf{1 , 2 1 2}$

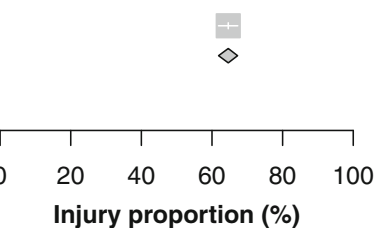

Fig. 5 Pooled injury proportions (\%) with $95 \%$ confidence intervals (CIs) of participants with time-loss injuries during a 1-year follow-up/ recall period. $P C$ prospective cohort study, $R C$ retrospective cohort study

injury proportion was lowest in cross-country runners, at $3.2 \%$ (95\% CI 0.9-10.5). One study that followed novice runners for a year reported a time-loss injury proportion of $27.3 \%$ (95\% CI 24.5-30.3). One study retrospectively assessed 1-year time-loss injury occurrence in recreational runners $[55.0 \% \quad(95 \% \mathrm{CI}$ 48.1-61.8)]. In long-distance road runners and marathon runners, the pooled injury proportions were $31.7 \%$ (95\% CI 25.8-38.2) and 52.0\% (95\% CI 43.1-60.8), respectively. One study reported a 1-year time-loss injury proportion of $64.6 \%$ (95 \% CI 61.9-67.2) among ultramarathon runners. The sensitivity analysis led to small differences in sprinters [62.8\% (95\% CI 54.4-70.4)], middle-distance runners [76.9\% (95\% CI 47.8-92.4)], cross-country runners [4.2\% (95\% CI $0.6-23.9)]$ and long-distance road runners [28.7\% (95\% CI 27.4-30.0)]. The injury proportions in novice and recreational runners were identical, and there were no studies in ultra-marathon runners (see Electronic Supplementary Material Appendix S5). 
Study

Design Injuries Participants

Population $=$ Novice runners

Bovens et al. (1989) [81]

Random effects model

Heterogeneity: not applicable for a single study

Population $=$ Cross-country runners

Reinking (2006)

Reinking et al. (2010) [51]

Random effects model

Heterogeneity: $P^{2}=57.8 \%, \tau^{2}=0.2091, p=0.1236$

Population = Road: long distance runners

Jacobs and Berson (1986) [55]

Lloyd et al. (1986) [56]

Koplan et al. (1995) [3]

Random effects model

Heterogeneity: $P^{R}=93.9 \%, \tau^{2}=0.1612, p<0.0001$

Population $=$ Marathon runners

Hölmich et al. (1989) [66]

Random effects model

Heterogeneity: not applicable for a single study

$571 \quad 1,246$

$\mathrm{RC}$

410

410

$\begin{array}{lrr}\text { RC } & 210 & 451 \\ \text { RC } & 80 & 260 \\ \text { RC } & 281 & 535\end{array}$

1,310

1,310
73

73

18
125

143
Injury proportion (\%)

$95 \% \mathrm{Cl}$

84.9 [74.6, 92.2]

$84.9[74.8,91.5]$

$66.7[41.0,86.7]$

$82.4[74.6,88.6]$

$77.4[60.6,88.4]$

$46.6[41.9,51.3]$

30.8 [25.2, 36.8]

$52.5[48.2,56.8]$

$43.2[32.2,54.9]$

$31.3[28.8,33.9]$

31.3 [28.8, 33.9]

Fig. 6 Pooled injury proportions (\%) with $95 \%$ confidence intervals (CIs) of participants with time-loss injuries during a follow-up/recall period of $>1$ year. $P C$ prospective cohort study, $R C$ retrospective cohort study

\subsubsection{Time-Loss Injuries During a Long Follow-Up/Recall Period}

Results were pooled from seven studies in which time-loss injuries during a long follow-up/recall period were monitored (Fig. 6) [3, 6, 51, 55, 56, 66, 81]. No studies were conducted among sprinting or middle- and long-distance track runners. One study prospectively followed novice runners during an 18-month running programme and reported an injury proportion of $84.9 \%$ (95 \% CI 74.8-91.5) [81]. Recreational runners were not studied over periods longer than a year. The pooled injury proportions in crosscountry runners and long-distance road runners were $77.4 \% \quad(95 \% \mathrm{CI} \quad 60.6-88.4)$ and $43.2 \% \quad(95 \% \mathrm{CI}$ $32.2-54.9)$, respectively. One study of marathon runners reported an injury proportion of $31.3 \% \quad(95 \% \mathrm{CI}$ 28.8-33.9) [66]. In the sensitivity analysis, three studies were removed from the analysis (see Electronic Supplementary Material Appendix S5) [55, 56, 66]. This increased the injury proportion among long-distance road runners to $52.5 \%$ (95\% CI 48.3-56.7), and no information on marathon runners was available.

\subsection{Anatomical Locations of Injuries}

The site-specific injury proportions can be found in Electronic Supplementary Material Appendix S5. An overview of the site-specific time-loss injury proportions can be found in Fig. 7. The numbers of injuries sustained in the hip/pelvis region were similar for all populations of runners, with injury proportions ranging from $5.7 \%$ in crosscountry runners to $10.8 \%$ in sprinting track athletes. Injury proportions in the upper leg were small for most populations of runners $(5.5-9.0 \%)$. In sprinting athletes, however, most injuries (32.9\%) occurred in the upper leg. The opposite was found for the knee region. Sprinters had the smallest number of injuries in the knee $(1.3 \%)$, while the injury proportions in the other populations varied from $22.5 \%$ (in cross-country runners) to $30.6 \%$ (in novice runners). Most injuries in recreational runners were reported around the knee $(26.3 \%)$. Novice runners $(34.7 \%)$, cross-country runners $(30.3 \%)$ and marathon runners $(29.9 \%)$ reported the most injuries in the lower leg. Sprinting athletes did not report ankle injuries; this range varied from $7.8 \%$ (in recreational runners) to $16.2 \%$ (in cross-country runners) in the other populations. Novice runners and sprinters reported foot injury proportions of 3.5 and $4.0 \%$, respectively. For cross-country runners $(8.1 \%)$, recreational runners $(10.1 \%)$ and marathon runners $(13.1 \%)$, the numbers of injuries in the foot were greater.

\section{Discussion}

This is the first systematic review to examine differences in injury proportions between different populations of runners. By pooling injury proportions according to follow-up/ recall periods and injury definitions (i.e. time-loss injuries, 


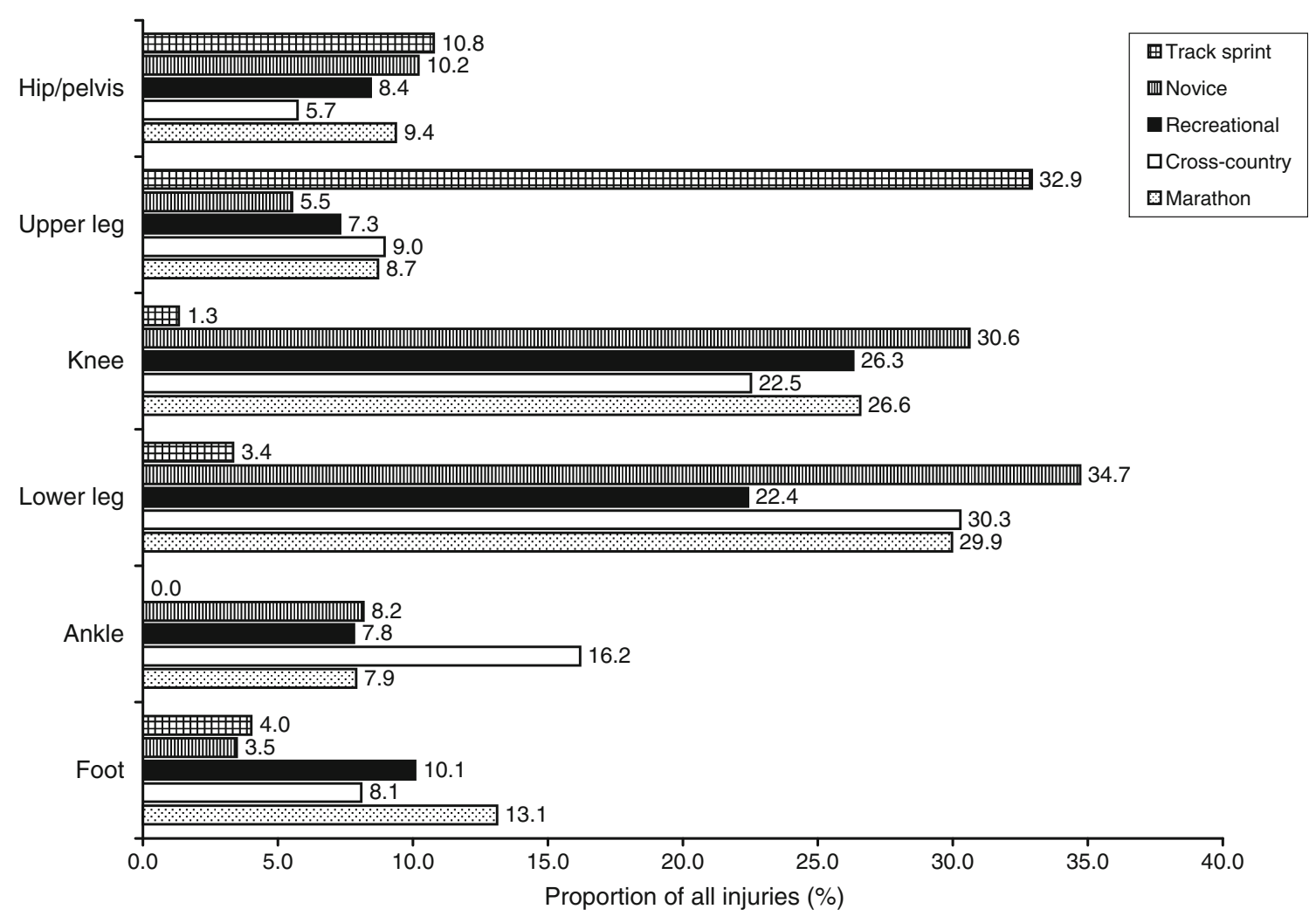

Fig. 7 Site-specific time-loss injury proportions (\%); injuries during events were excluded. It should be noted that the sum of the injury proportions is not equal to $100 \%$, because a few injuries could not be

medical-attention injuries and pain-related injuries) for different populations of runners, we organized the results reported in the literature. Medical-attention injuries were mainly monitored during running events. Except in ultramarathon runners, the numbers of medical-attention injuries were small in all populations of runners. A few studies monitored pain-related injuries. The highest injury proportion found in this review $(94.4 \%)$ was for painrelated injuries among cross-country runners during a lifetime recall period [6]. The lowest injury proportion was found for medical-attention injuries in long-distance runners during an event [60]. Depending on the duration of follow-up/recall, the proportion of time-loss injuries was highest in short-distance track runners (middle-distance runners and sprinters) and ultra-marathon runners.

The numbers of medical-attention injuries during an event varied considerably between different populations of runners. During shorter road races, only $0.9 \%$ of participants requested medical attention at a first-aid station. This percentage was small compared with those in marathon runners $(7.8 \%)$ and ultra-marathon runners $(65.5 \%)$. This increase in medical attention with increasing running distance could also be seen in track athletes. Only $7.2 \%$ of sprinting athletes required medical attention, while this classified within these anatomical locations (see Electronic Supplementary Material Appendix S6)

proportion increased to $15.6 \%$ in long-distance track runners. This discrepancy in injury proportions might have been caused by differences in the accessibility of medical facilities; lack of accessibility is a drawback with regard to registering all problems that require medical attention [98, 99]. This may explain the larger number of medical-attention injuries in marathon runners compared with longdistance road runners, since the number of first-aid stations during a marathon race is relatively large. In a multi-day ultra-race event, runners often had to notify the medical staff about possible problems several times a day. It is likely that this high accessibility of medical services led to the enormous proportion of medical problems reported during ultra-race events. Moreover, accessibility to medical facilities is also dependent on the level of sports participation. For instance, the studies in which medical attention for track runners was registered during an event were all performed in elite athletes during international championships. These athletes have direct access to clinicians, making it impossible to generalize these injury proportions to lower-level track runners. As a result, the reporting of medical-attention injuries does not necessarily reflect the number of injuries sustained in a cohort of runners. Therefore, this method is less suitable for making 
statements about the number of injuries sustained in a group of runners.

A few studies among marathon runners examined the number of time-loss injuries sustained during an event. The pooled estimate of time-loss injuries during a marathon race was $20.6 \%$, which is considerably greater than the number of medical-attention injuries reported during a marathon event $(7.2 \%)$. Also, $73.9 \%$ of the marathon runners reported pain-related injuries during a race. These differences emphasize that medical-attention injuries do not reflect the number of complaints sustained during an event.

For capturing running-related complaints sustained during a given period of time, time-loss injuries were often registered. When looking at studies with a short follow-up/ recall period, no large differences in time-loss injury occurrence existed between novice runners $(26.4 \%)$, recreational runners $(28.0 \%)$ and cross-country runners (19.7\%). Marathon runners, on the other hand, reported more time-loss injuries during these short periods $(64.7 \%)$. These findings suggest that the injury risk is greater for runners training for longer distances. This hypothesis is partly supported by the results from the time-loss injury proportions during a 1-year period. However, middle-distance track runners and sprinters reported high injury proportions too (63.9 and $63.8 \%$, respectively). Hence, a U-shaped pattern between the running distance and the injury risk may exist. By contrast, the injury proportion reported in cross-country runners was remarkably small $(3.2 \%)$. In several studies, injury occurrence was monitored in high school athletes throughout the year [22, 24, 26]. In these studies, injury proportions among cross-country runners varied from 1.6 to $10.6 \%$, which is relatively small compared with the injury proportion of $19.7 \%$ reported during a single season. One study retrospectively assessed pain-related injuries in cross-country runners over a 1-year period [53], finding an injury proportion of $72.7 \%$, which indicates that many cross-country athletes suffer from injuries. The exact reason for the small number of time-loss injuries reported among cross-country runners is unknown, but may have to do with the methods of injury surveillance used in the studies among high school athletes. In those studies, injuries were monitored by the coach, who subsequently reported the injuries to the researchers.

In contrast with the studies that had a short or 1-year follow-up/recall period, studies that used a longer period of time displayed an opposite relation between injury proportions and running distance. In these studies, novice runners had the highest injury proportion (84.9\%), followed by cross-country runners $(77.4 \%)$. Marathon runners, on the other hand, reported the lowest injury proportions $(31.3 \%)$. One notable difference between the study in novice runners and the other studies was its prospective character [81]. More injuries are likely to be registered in a prospective cohort study with a longer follow-up period than in a retrospective study with a similar recall period. It should also be noted that the differences between the populations decreased in the sensitivity analysis, in which only studies with a low ROB were included. This may be an indication that the opposite trend was caused by the small number of high-quality studies that assessed injuries over a longer period of time. After all, only seven studies examined injury occurrence over the long term, and only four of them were suitable for the sensitivity analysis (ROB score $\geq 50$ ).

In a small number of studies, anatomical regions were registered where the injury occurred. Admittedly, only those studies focusing on all injuries were included in this analysis. The results of the studies showed that in recreational runners, most injuries occurred at the knee, while lower leg injuries were more common in novice, crosscountry and marathon runners. This is in line with the review by Lopes et al., which showed that most running injuries were located at the foot, ankle, lower leg and knee [8]. The distribution of injuries across sprinting athletes, however, was notably different: most of their injuries were sustained in the upper leg, followed by the hip/pelvis. During normal running, propulsion is achieved mainly by the structures of the lower leg [100], but during running at high speeds (i.e. sprinting), propulsion is more dependent on power generated at the hip. This is achieved by increasing the demand on the upper leg muscles, resulting in a greater biomechanical load in these structures [100]. This may explain the different injury distribution in sprinting athletes compared with other populations of runners.

It is worth noting that the smallest number of studies involved recreational runners-supposedly the largest group of runners worldwide [95]. This may have to do with the definition of recreational runners that was used in this review (non-competitive runners, or runners participating in road races shorter than $10 \mathrm{~km}$ ). It is also plausible that this population of runners is ignored for practical reasons. Running events or organized running groups are often used to approach runners for inclusion in a study. The non-organized nature of recreational running makes it difficult to target these runners and include them in a study.

In the literature, it is often assumed that novice runners have a higher injury risk than more experienced runners [7, 82, 101]. The results of this systematic review do not support this assumption, instead giving an indication that the running distance and the injury risk follow a U-shaped pattern, in which short-distance track runners and ultramarathon runners have the highest injury risk. Unfortunately, most studies included in this review only reported injury proportions over a given period of time. For comparative reasons, it would be better to relate the injury risk to the amount of time spent running [68] — for instance, 
expressing the number of injuries as a density per $1000 \mathrm{~h}$ of running. A number of studies reported injury occurrence in terms of running exposure [7, 27, 42, 44, 49, 78, 83, 88, 95]; their results showed enormous differences between novice runners (33 injuries per $1000 \mathrm{~h}$ of running [83]) and middledistance track runners (5.6 injuries per $1000 \mathrm{~h}$ of running [27]). In addition, most studies included in this systematic review did not take censoring into account. Censoring, however, may cause an underestimation of the injury risk in a population of runners. This is particularly true when the cumulative incidence proportion is related to the amount of time spent running. Then, participants who spent less time running would be censored even when they successfully completed the study. In such a case, censoring would result in an increased cumulative injury proportion. In the study by Nielsen et al. among novice runners, an overall injury proportion of $27.3 \%$ was found. However, when censoring was taken into account, the cumulative injury proportion after $500 \mathrm{~km}$ of running was almost $50 \%$ [25].

\subsection{Limitations}

Some limitations of this review should be mentioned. First, only studies written in English were included in the review. Possibly, relevant articles written in other languages were missed. Second, the purpose of this study was to compare injury proportions between different populations of runners. To this end, we identified nine different populations that, in our opinion, discriminated in terms of running experience and running distance. However, the level of running participation was not taken into account. For instance, the studies in track runners included runners participating in world championships, as well as recreational track runners. Third, only studies reporting data in one or more homogeneous populations of runners separately were included in this review. It is therefore possible that some studies were not included, as a result of our defined populations. In addition, the populations might have overlapped; however, by clearly describing our populations, we tried to address this issue. Fourth, injury proportions from studies using a similar injury definition and with a comparable follow-up/recall period were pooled. Definitions were categorized into pain-related injuries, medical-attention injuries and time-loss injuries. There were small differences within injury definitions classified in the same category, which might have influenced the injury proportions reported in these studies. Finally, in this review, both retrospective and prospective cohort studies were pooled. The number of injuries reported in a study is dependent on the study design that is used. It is, however, plausible to assume that this effect becomes more apparent in studies with longer follow-up or recall periods, because of recall bias. In the current review, no distinct differences in injury proportions were observed between prospective and retrospective studies. For this reason, the results of both study designs were pooled. The heterogeneity of the pooled studies was high. This could have been the result of pooling both retrospective and prospective cohort studies. Exclusion of retrospective cohort studies from the metaanalysis did not result in less heterogeneity though. This indicates that the heterogeneity was caused by other differences between studies. Besides differences in populations, study designs and injury definitions, heterogeneity could have been caused by differences in injury assessment. In some studies, injuries were diagnosed by a medical professional, while other injury reports were based on self-reports. Differences in demographics within a population could also lead to heterogeneity. Because injury proportions were the outcome of interest in the current review, a meta-analysis using a random-effects model was used. Consequently, heterogeneity between studies was allowed.

\section{Conclusions}

The numbers of medical-attention injuries during an event were small for most populations of runners, except for ultra-marathon runners, $65.6 \%$ of whom reported medicalattention injuries during a multi-day running event. Large differences in time-loss injury proportions between different populations of runners existed, ranging from a pooled estimate for cross-country runners of $3.2 \%$ to an injury proportion of $84.9 \%$ in novice runners. Injury proportions were affected by the duration of follow-up/recall. Overall, however, a U-shaped pattern between the running distance and the time-loss injury proportion seemed to exist, in which sprinting athletes and ultra-marathon runners had the highest proportions of time-loss injuries. Relatively few studies reported the injury incidence in relation to the amount of time spent running. Future prospective studies of injury surveillance are therefore highly recommended to take running exposure and censoring into account.

Acknowledgments This review was funded by a grant from the Netherlands Organisation for Health Research and Development (ZonMw; Grant Number 50-50305-98-12001). The authors have no competing interests. The authors would like to thank Truus van Ittersum for her assistance in developing the search strategy for this systematic review.

Open Access This article is distributed under the terms of the Creative Commons Attribution 4.0 International License (http:// creativecommons.org/licenses/by/4.0/), which permits unrestricted use, distribution, and reproduction in any medium, provided you give appropriate credit to the original author(s) and the source, provide a link to the Creative Commons license, and indicate if changes were made. 


\section{References}

1. Saragiotto BT, Yamato TP, Hespanhol Junior LC, et al. What are the main risk factors for running-related injuries? Sports Med. 2014;44:1153-63.

2. Nielsen RO, Rønnow L, Rasmussen S, et al. A prospective study on time to recovery in 254 injured novice runners. PloS one. 2014;9:e99877.

3. Koplan JP, Rothenberg RB, Jones EL. The natural history of exercise: a 10-yr follow-up of a cohort of runners. Med Sci Sports Exerc. 1995;27:1180-4.

4. Kluitenberg B, van Middelkoop M, Smits DW, et al. The NLstart2run study: incidence and risk factors of running-related injuries in novice runners. Scand J Med Sci Sports. 2014; doi:10.1111/sms.12346 (Epub 2014 Dec 1).

5. Chandy TA, Grana WA. Secondary school athletic injury in boys and girls: a three-year comparison. Phys Sports Med. 1985;13:106-11.

6. Reinking MF. Exercise-related leg pain in female collegiate athletes: the influence of intrinsic and extrinsic factors. Am J Sports Med. 2006;34:1500-7.

7. Buist I, Bredeweg S, Bessem B, et al. Incidence and risk factors of running-related injuries during preparation for a 4-mile recreational running event. Br J Sports Med. 2010;44:598-604.

8. Lopes AD, Hespanhol Junior LC, Yeung SS, et al. What are the main running-related musculoskeletal injuries? A systematic review. Sports Med. 2012;42:891-905.

9. van Mechelen W, Hlobil H, Kemper HC. Incidence, severity, aetiology and prevention of sports injuries: a review of concepts. Sports Med. 1992;14:82-99.

10. van der Windt DA, Thomas E, Pope DP, et al. Occupational risk factors for shoulder pain: a systematic review. Occup Environ Med. 2000;57:433-42.

11. Nielsen RO, Buist I, Sorensen H, et al. Training errors and running related injuries: a systematic review. Int J Sports Phys Ther. 2012;7:58-75.

12. $\mathrm{R}$ Core Team. R: a language and environment for statistical computing. R Foundation for Statistical Computing, 2014. http://www.R-project.org. Accessed 21 Jan 2015.

13. Schwarzer G. Meta: meta-analysis with $\mathrm{R}$ : $\mathrm{R}$ package version 4.0.2. R Foundation for Statistical Computing, 2014. http:// CRAN.R-project.org/package=meta. Accessed 21 Jan 2015.

14. Higgins JPT, Green S. Cochrane handbook for systematic reviews of interventions: version 5.1.0 (updated March 2011). The Cochrane Collaboration, 2011. http://handbook.cochrane.org/. Accessed 21 Jan 2015.

15. van Gent RN, Siem D, van Middelkoop M, et al. Incidence and determinants of lower extremity running injuries in long distance runners: a systematic review. $\mathrm{Br} \mathrm{J}$ Sports Med. 2007;41:469-80.

16. Beachy G, Akau CK, Martinson M, et al. High school sports injuries a longitudinal study at Punahou School: 1988 to 1996. Am J Sports Med. 1997;25:675-81.

17. Bennell KL, Malcolm SA, Thomas SA, et al. Risk factors for stress fractures in female track-and-field athletes: a retrospective analysis. Clin J Sport Med. 1995;5:229-35.

18. D'Souza D. Track and field athletics injuries-a one-year survey. Br J Sports Med. 1994;28:197-202.

19. Eickhoff CA, Hossain SA, Slawski DP. Effects of prescribed foot orthoses on medial tibial stress syndrome in collegiate cross-country runners. Clin Kinesiol. 2000;54:76-80.

20. Garrick JG, Requa RK. Injuries in high school sports. Pediatrics. 1978;61:465-9.

21. Garrick JG, Requa RK. Girls' sports injuries in high school athletics. JAMA. 1978;239:2245-8.
22. Grana WA. Summary of 1978-79 injury registry for Oklahoma secondary schools. J Okla State Med Assoc. 1979;72:369-72.

23. Koplan JP, Powell KE, Sikes RK, et al. An epidemiologic study of the benefits and risks of running. JAMA. 1982;248:3118-21.

24. McLain LG, Reynolds S. Sports injuries in a high school. Pediatrics. 1989;84:446-50.

25. Nielsen RO, Buist I, Parner ET, et al. Predictors of running-related injuries among 930 novice runners: a 1-year prospective followup study. Orthop J Sports Med. 2013;1(1):2325967113487316. doi:10.1177/2325967113487316.

26. Shively RA, Grana WA, Ellis D. High school sports injuries. Phys Sportsmed. 1981;9:46-50.

27. Lysholm J, Wiklander J. Injuries in runners. Am J Sports Med. 1987;15:168-71.

28. Bennell KL, Malcolm SA, Thomas SA, et al. The incidence and distribution of stress fractures in competitive track and field athletes: a twelve-month prospective study. Am J Sports Med. 1996;24:211-7.

29. Alonso JM, Junge A, Renström $P$, et al. Sports injuries surveillance during the 2007 IAAF World Athletics Championships. Clin J Sport Med. 2009;19:26-32.

30. Alonso JM, Tscholl PM, Engebretsen L, et al. Occurrence of injuries and illnesses during the 2009 IAAF World Athletics Championships. Br J Sports Med. 2010;44:1100-5.

31. Alonso JM, Edouard P, Fischetto G, et al. Determination of future prevention strategies in elite track and field: analysis of Daegu 2011 IAAF Championships injuries and illnesses surveillance. Br J Sports Med. 2012;46:505-14.

32. Longo UG, Rittweger J, Garau G, et al. No influence of age, gender, weight, height, and impact profile in achilles tendinopathy in masters track and field athletes. Am J Sports Med. 2009;37:1400-5.

33. Fredericson M, Ngo J, Cobb K. Effects of ball sports on future risk of stress fracture in runners. Clin $\mathrm{J}$ Sport Med. 2005;15:136-41.

34. Nicholl JP, Williams BT. Medical problems before and after a popular marathon. Br Med J (Clin Res Ed). 1982;285:1465-6.

35. Nicholl JP, Williams BT. Injuries sustained by runners during a popular marathon. Br J Sports Med. 1983;17:10-5.

36. Yeung SS, Yeung EW, Wong TW. Provision of physiotherapy at the Tsing Ma Bridge international marathon and $10 \mathrm{~km}$ race in Hong Kong. Br J Sports Med. 1998;32:336-7.

37. Micklesfield LK, Hugo J, Johnson C, et al. Factors associated with menstrual dysfunction and self-reported bone stress injuries in female runners in the ultra- and half-marathons of the Two Oceans. Br J Sports Med. 2007;41:679-83.

38. Chang WL, Shih YF, Chen WY. Running injuries and associated factors in participants of ING Taipei Marathon. Phys Ther Sport. 2012;13:170-4.

39. Sugiura Y, Saito T, Sakuraba K, et al. Strength deficits identified with concentric action of the hip extensors and eccentric action of the hamstrings predispose to hamstring injury in elite sprinters. J Orthop Sports Phys Ther. 2008;38:457-64.

40. Yeung SS, Suen AM, Yeung EW. A prospective cohort study of hamstring injuries in competitive sprinters: preseason muscle imbalance as a possible risk factor. $\mathrm{Br} \mathrm{J}$ Sports Med. 2009;43:589-94.

41. Jacobsson J, Timpka T, Kowalski J, et al. Injury patterns in Swedish elite athletics: annual incidence, injury types and risk factors. Br J Sports Med. 2013;47:941-52.

42. Rauh MJ, Margherita AJ, Rice SG, et al. High school cross country running injuries: a longitudinal study. Clin J Sport Med. 2000;10:110-6.

43. Bennett JE, Reinking MF, Pluemer B, et al. Factors contributing to the development of medial tibial stress syndrome in high school runners. J Orthop Sports Phys Ther. 2001;31:504-10. 
44. Rauh MJ, Koepsell TD, Rivara FP, et al. Epidemiology of musculoskeletal injuries among high school cross-country runners. Am J Epidemiol. 2006;163:151-9.

45. Rauh MJ, Koepsell TD, Rivara FP, et al. Quadriceps angle and risk of injury among high school cross-country runners. J Orthop Sports Phys Ther. 2007;37:725-33.

46. Reinking MF, Hayes AM. Intrinsic factors associated with exercise-related leg pain in collegiate cross-country runners. Clin J Sport Med. 2006;16:10-4.

47. Kelsey JL, Bachrach LK, Procter-Gray E, et al. Risk factors for stress fracture among young female cross-country runners. Med Sci Sports Exerc. 2007;39:1457-63.

48. Laker SR, Saint-Phard D, Tyburski M, et al. Stress fractures in elite cross-country athletes. Orthopedics. 2007;30:313-5.

49. Plisky MS, Rauh MJ, Heiderscheit B, et al. Medial tibial stress syndrome in high school cross-country runners: incidence and risk factors. J Orthop Sports Phys Ther. 2007;37:40-7.

50. Reinking MF, Austin TM, Hayes AM. Exercise-related leg pain in collegiate cross-country athletes: extrinsic and intrinsic risk factors. J Orthop Sports Phys Ther. 2007;37:670-8.

51. Reinking MF, Austin TM, Hayes AM. Risk factors for selfreported exercise-related leg pain in high school cross-country athletes. J Athl Train. 2010;45:51-7.

52. Finnoff JT, Hall MM, Kyle K, et al. Hip strength and knee pain in high school runners: a prospective study. PM R. 2011;3:792-801.

53. Bennett JE, Reinking MF, Rauh MJ. The relationship between isotonic plantar flexor endurance, navicular drop, and exerciserelated leg pain in a cohort of collegiate cross-country runners. Int J Sports Phys Ther. 2012;7:267-78.

54. Hughes WA, Noble HB, Porter M. Distance race injuries: an analysis of runners' perceptions. Phys Sportsmed. 1985;13:43-58.

55. Jacobs SJ, Berson BL. Injuries to runners: a study of entrants to a 10,000 meter race. Am J Sports Med. 1986;14:151-5.

56. Lloyd T, Triantafyllou SJ, Baker ER, et al. Women athletes with menstrual irregularity have increased musculoskeletal injuries. Med Sci Sports Exerc. 1986;18:374-9.

57. Marti B, Vader JP, Minder CE, et al. On the epidemiology of running injuries. The Bern Grand-Prix study. Am J Sports Med. 1984;1988(16):285-94.

58. Marti B. Benefits and risks of running among women: an epidemiologic study. Int J Sports Med. 1988;9:92-8.

59. Woolf SK, Barfield WR, Nietert PJ, et al. The Cooper River Bridge Run Study of low back pain in runners and walkers. J South Orthop Assoc. 2002;11:136-43.

60. Pasquina PF, Griffin SC, Anderson-Barnes VC, et al. Analysis of injuries from the Army Ten Miler: a 6-year retrospective review. Mil Med. 2013;178:55-60.

61. Caldwell J. Experience from the 1980 Midnight Sun Marathon: injuries and training. Alaska Med. 1981;23:18-21.

62. Maughan RJ, Miller JD. Incidence of training-related injuries among marathon runners. Br J Sports Med. 1983;17:162-5.

63. Kretsch A, Grogan R, Duras P, et al. Melbourne marathon study. Med J Aust. 1980;1984(141):809-14.

64. McKelvie SJ, Valliant PM, Asu ME. Physical training and personality factors as predictors of marathon time and training injury. Percept Mot Skills. 1985;60:551-66.

65. Hölmich P, Darre E, Jahnsen F, et al. The elite marathon runner: problems during and after competition. Br J Sports Med. 1988;22:19-21.

66. Hölmich P, Christensen SW, Darre E, et al. Non-elite marathon runners: health, training and injuries. $\mathrm{Br} \mathrm{J}$ Sports Med. 1989;23:177-8.

67. Macera CA, Pate RR, Woods J, et al. Postrace morbidity among runners. Am J Prev Med. 1991;7:194-8.
68. Jakobsen BW, Krøner K, Schmidt SA, et al. Prevention of injuries in long-distance runners. Knee Surg Sports Traumatol Arthrosc. 1994;2:245-9.

69. Satterthwaite P, Larmer P, Gardiner J, et al. Incidence of injuries and other health problems in the Auckland Citibank Marathon, 1993. Br J Sports Med. 1996;30:324-6.

70. Roberts WO. A 12-yr profile of medical injury and illness for the Twin Cities Marathon. Med Sci Sports Exerc. 2000;32:1549-55.

71. Van Middelkoop M, Kolkman J, Van Ochten J, et al. Prevalence and incidence of lower extremity injuries in male marathon runners. Scand J Med Sci Sports. 2008;18:140-4.

72. van Middelkoop M, Kolkman J, van Ochten J, et al. Risk factors for lower extremity injuries among male marathon runners. Scand J Med Sci Sports. 2008;18:691-7.

73. Parker DT, Weitzenberg TW, Amey AL, et al. Group training programs and self-reported injury risk in female marathoners. Clin J Sport Med. 2011;21:499-507.

74. Rasmussen $\mathrm{CH}$, Nielsen RO, Juul MS, et al. Weekly running volume and risk of running-related injuries among marathon runners. Int J Sports Phys Ther. 2013;8:111-20.

75. Hutson MA. Medical implications of ultra marathon running: observations on a six day track race. $\mathrm{Br} \mathrm{J}$ Sports Med. 1984;18:44-5.

76. Fallon KE. Musculoskeletal injuries in the ultramarathon: the 1990 Westfield Sydney to Melbourne Run. Br J Sports Med. 1996;30:319-23.

77. Bishop GW, Fallon KE. Musculoskeletal injuries in a six-day track race: ultramarathoner's ankle. Clin J Sport Med. 1999;9:216-20.

78. Krabak BJ, Waite B, Schiff MA. Study of injury and illness rates in multiday ultramarathon runners. Med Sci Sports Exerc. 2011;43:2314-20.

79. Scheer BV, Murray A. Al Andalus Ultra Trail: an observation of medical interventions during a 219-km, 5-day ultramarathon stage race. Clin J Sport Med. 2011;21:444-6.

80. Hoffman MD, Krishnan E. Health and exercise-related medical issues among 1,212 ultramarathon runners: baseline findings from the Ultrarunners Longitudinal TRAcking (ULTRA) Study. PloS One. 2014;9:e83867.

81. Bovens AM, Janssen GM, Vermeer HG, et al. Occurrence of running injuries in adults following a supervised training program. Int J Sports Med. 1989;10(Suppl 3):S186-90.

82. Buist I, Bredeweg SW, van Mechelen W, et al. No effect of a graded training program on the number of running-related injuries in novice runners: a randomized controlled trial. Am J Sports Med. 2008;36:33-9.

83. Buist I, Bredeweg SW, Lemmink KAPM, et al. Predictors of running-related injuries in novice runners enrolled in a systematic training program: a prospective cohort study. Am J Sports Med. 2010;38:273-80.

84. Thijs Y, De Clercq D, Roosen P, et al. Gait-related intrinsic risk factors for patellofemoral pain in novice recreational runners. $\mathrm{Br}$ J Sports Med. 2008;42:466-71.

85. Ghani Zadeh Hesar N, Van Ginckel A, Cools A, et al. A prospective study on gait-related intrinsic risk factors for lower leg overuse injuries. Br J Sports Med. 2009;43:1057-61.

86. Van Ginckel A, Thijs Y, Hesar NG, et al. Intrinsic gait-related risk factors for Achilles tendinopathy in novice runners: a prospective study. Gait Posture. 2009;29:387-91.

87. Thijs Y, Pattyn E, Van Tiggelen D, et al. Is hip muscle weakness a predisposing factor for patellofemoral pain in female novice runners? A prospective study. Am J Sports Med. 2011;39:1877-82.

88. Bredeweg SW, Zijlstra S, Bessem B, et al. The effectiveness of a preconditioning programme on preventing running-related injuries in novice runners: a randomised controlled trial. $\mathrm{Br} \mathrm{J}$ Sports Med. 2012;46:865-70. 
89. Bredeweg SW, Kluitenberg B, Bessem B, et al. Differences in kinetic variables between injured and noninjured novice runners: a prospective cohort study. J Sci Med Sport. 2013;16:205-10.

90. Bredeweg SW, Buist I, Kluitenberg B. Differences in kinetic asymmetry between injured and noninjured novice runners: a prospective cohort study. Gait Posture. 2013;38:847-52.

91. Nielsen RO, Buist I, Parner ET, et al. Foot pronation is not associated with increased injury risk in novice runners wearing a neutral shoe: a 1-year prospective cohort study. Br J Sports Med. 2013;48:440-7.

92. Nielsen RO, Cederholm P, Buist I, et al. Can GPS be used to detect deleterious progression in training volume among runners? J Strength Cond Res. 2013;27:1471-8.

93. Lopes AD, Costa LO, Saragiotto BT, et al. Musculoskeletal pain is prevalent among recreational runners who are about to compete: an observational study of 1049 runners. J Physiother. 2011;57:179-82.

94. Hespanhol Junior LC, Costa LO, Carvalho AC, et al. A description of training characteristics and its association with previous musculoskeletal injuries in recreational runners: a cross-sectional study. Rev Bras Fisioter. 2012;16:46-53.

95. Hespanhol Junior LC, Pena Costa LO, Lopes AD. Previous injuries and some training characteristics predict running-related injuries in recreational runners: a prospective cohort study. J Physiother. 2013;59:263-9.

96. Satterthwaite P, Norton R, Larmer P, et al. Risk factors for injuries and other health problems sustained in a marathon. $\mathrm{Br} \mathrm{J}$ Sports Med. 1999;33:22-6.

97. Ogwumike OO, Adeniyi AF. The SPLASH/ICPC integrity marathon in Ibadan, Nigeria: incidence and management of injuries and marathon-related health problems. BMC Sports Sci Med Rehabil. 2013;5:1-5.

98. Clarsen B, Bahr R. Matching the choice of injury/illness definition to study setting, purpose and design: one size does not fit all! Br J Sports Med. 2014;48:510-2.

99. Orchard JW, Newman D, Stretch R, et al. Methods for injury surveillance in international cricket. $\mathrm{Br} \mathrm{J}$ Sports Med. 2005;39:e22.

100. Schache AG, Dorn TW, Williams GP, et al. Lower-limb muscular strategies for increasing running speed. J Orthop Sports Phys Ther. 2014;44:813-24.

101. Tonoli C, Cumps E, Aerts I, et al. Incidence, risk factors and prevention of running related injuries in long-distance running: a systematic review. Sport Geneeskd. 2010;43:12-8. 